\title{
NICHOLS ALGEBRAS WITH MANY CUBIC RELATIONS
}

\author{
I. HECKENBERGER, A. LOCHMANN, AND L. VENDRAMIN
}

\begin{abstract}
Nichols algebras of group type with many cubic relations are classified under a technical assumption on the structure of Hurwitz orbits of the third power of the underlying indecomposable rack. All such Nichols algebras are finite-dimensional, and their Hilbert series have a factorization into quantum integers. Also, all known finite-dimensional elementary Nichols algebras turn out to have many cubic relations. The technical assumption of our theorem can be removed if a conjecture in the theory of cellular automata can be proven.
\end{abstract}

\section{Contents}

1. Introduction

2. Racks

3. On the cycle structure of racks

4. Quotients of Hurwitz orbits

5. Cellular automata over homogeneous spaces $\quad 6325$

6. Plagues on Hurwitz orbits 6328

7. Proof of Theorem 6.3 6329

8. Nichols algebras with many cubic relations $\quad 6350$

9. Computation of the 2-cocycles and the main theorem $\quad 6353$

Acknowledgements $\quad 6355$

References 6355

\section{INTRODUCTION}

Let $(V, c)$ be a braided vector space. A fundamental question in the theory of Hopf algebras is whether the Nichols algebra $\mathfrak{B}(V)$ (which heavily depends on $c$ ) is finite-dimensional. If $V$ is of diagonal type, then $\mathfrak{B}(V)$ is known to have a restricted $\mathrm{PBW}$ basis and the PBW generators are parametrized by Lyndon words. The multidegrees of these Lyndon words can be regarded as positive roots in a generalized root system which has the symmetry of a Weyl groupoid. Moreover, the existence of the restricted PBW basis implies that the Hilbert series of $\mathfrak{B}(V)$ is rational.

Received by the editors February 3, 2013 and, in revised form, June 25, 2013.

2010 Mathematics Subject Classification. Primary 16T05; Secondary 20F99, 16P90.

The first author was supported by the German Research Foundation via a Heisenberg professorship.

The third author was supported by CONICET and the Alexander von Humboldt Foundation. 
If the braiding $c$ is of group type, then the structure of $\mathfrak{B}(V)$ is much less understood. Moreover, only a few finite-dimensional examples are known which are not of diagonal type. One common feature of them is the factorization of the Hilbert series of $\mathfrak{B}(V)$ as

$$
\mathcal{H}_{\mathfrak{B}(V)}(t)=\prod_{i=1}^{k_{1}}\left(a_{i}\right)_{t} \prod_{i=1}^{k_{2}}\left(b_{i}\right)_{t^{2}}
$$

for some $k_{1}, k_{2} \geq 0, a_{1}, \ldots, a_{k_{1}}, b_{1}, \ldots, b_{k_{2}} \geq 2$, where $(a)_{t^{b}}=1+t^{b}+t^{2 b}+\cdots+$ $t^{(a-1) b}$ for all $a, b \geq 1$. We say that $\mathcal{H}_{\mathfrak{B}(V)}(t)$ is t-integral of depth two. The $t$ integrality of all known Hilbert series of Nichols algebras of group type was the starting point of a classification program in [5] and [6]. It turned out that under additional technical restrictions the $t$-integrality of the Hilbert series is equivalent to an inequality on the dimensions of the homogeneous components of low degree of the Nichols algebra and that a complete list can be given.

Let $G$ be a group, $\mathbb{k}$ be a field and $V \in \mathbb{k}_{\mathbb{k} G}^{\mathbb{k} G} \mathcal{Y} \mathcal{D}$. Then the Nichols algebra $\mathfrak{B}(V)$ is called of group type. We say that the Nichols algebra $\mathfrak{B}(V)$ is elementary if $V$ is finite-dimensional, absolutely irreducible and its support

$$
\operatorname{supp} V=\left\{x \in G \mid V_{x} \neq 0\right\}
$$

generates the group $G$. A fundamental problem in the theory of Nichols algebras is the classification of finite-dimensional elementary Nichols algebras. In fact, often it is not important to know $G$. Let $H$ be a group. We say that two Yetter-Drinfeld modules $V \in \underset{\mathbb{k} G}{\mathbb{k} G} \mathcal{Y} \mathcal{D}, W \in \mathbb{k}_{\mathbb{k} H}^{\mathbb{k} H} \mathcal{Y} \mathcal{D}$ are bg-equivalen 11 if there exists a bijection $\varphi: \operatorname{supp} V \rightarrow \operatorname{supp} W$ and a linear isomorphism $\psi: V \rightarrow W$ such that

$$
\psi\left(V_{g}\right)=W_{\varphi(g)}, \quad \psi(g v)=\varphi(g) \psi(v)
$$

for all $g, x \in \operatorname{supp} V, v \in V$. Two Nichols algebras of group type are called $b g$ equivalent if their degree one parts are bg-equivalent. Then it is more convenient to ask for all finite-dimensional elementary Nichols algebras up to bg-equivalence. The answer to this problem is unknown to a large extent. However, there are indications that the following conjecture is possibly true:

Conjecture 1.1.2 All finite-dimensional elementary Nichols algebras are t-integral of depth two. In particular, any such Nichols algebra is bg-equivalent to one of those listed in Table 9.1 .

In this paper we extend the results in [5] and [6]. More precisely, we reduce the classification problem of elementary Nichols algebras with $t$-integral Hilbert series of depth two to a problem for cellular automata on braid group orbits.

Let us discuss some details of our approach. Assume first that the Hilbert series of $\mathfrak{B}(V)$ is $t$-integral of depth two. In [6, Section 2.1] it was shown that then the inequality

$$
\operatorname{dim} \operatorname{ker}\left(1+c_{12}+c_{12} c_{23}\right) \geq \frac{1}{3} \operatorname{dim} V\left((\operatorname{dim} V)^{2}-1\right)
$$

holds. Now, if this inequality holds for $V$, we say that $\mathfrak{B}(V)$ has many cubic relations. Our intention is to classify all Nichols algebras with many cubic relations

\footnotetext{
${ }^{1} \mathrm{~g}$ refers to the grading by the group, and b refers to the braiding.

${ }^{2}$ This conjecture was posed in the Oberwolfach mini-workshop "Nichols algebras and Weyl groupoids" in October 2012.
} 
and to prove that their Hilbert series are $t$-integral of depth two. If $\operatorname{supp} V$ is a braided rack, then this claim was proven in [6]. Here we attack the general problem.

Recall that the braid group $\mathbb{B}_{3}$ can be presented by generators $\sigma_{1}$ and $\sigma_{2}$ and relation $\sigma_{1} \sigma_{2} \sigma_{1}=\sigma_{2} \sigma_{1} \sigma_{2}$. The group $\mathbb{B}_{3}$ acts on $(\operatorname{supp} V)^{3}$ by $\sigma_{1} \cdot(x, y, z)=$ $(x \triangleright y, x, z)$ and $\sigma_{2} \cdot(x, y, z)=(x, y \triangleright z, y)$ for all $x, y, z \in \operatorname{supp} V$, where $\triangleright$ means conjugation. The orbits of the action of $\mathbb{B}_{3}$ are called Hurwitz orbits. We assume that the quotients of the Hurwitz orbits of $(\operatorname{supp} V)^{3}$ by the action of the center of the braid group have only $\sigma_{1}$-cycles of length $\leq 4$. (The braidedness condition on racks means that the Hurwitz orbits of $(\operatorname{supp} V)^{3}$ themselves have only $\sigma_{1}$-cycles of length $\leq 3$.) This way we cover the braided case and the cases $\operatorname{supp} V=\operatorname{Aff}(5, i)$ with $i=2,3$, for which finite-dimensional Nichols algebras are known to exist. On the other hand, we have to deal with infinitely many Hurwitz orbits in contrast to [6].

Looking at homogeneous components, dim $\operatorname{ker}\left(1+c_{12}+c_{12} c_{23}\right)$ can be estimated from above by a purely combinatorial way. Choose a subset $Y$ of $(\operatorname{supp} V)^{3}$ and take an element $\bar{\alpha} \in \oplus_{(x, y, z) \in Y} V_{x} \otimes V_{y} \otimes V_{z}$. Let $k(Y, \bar{\alpha})$ be the set of all $\alpha \in$ ker $\left(1+c_{12}+c_{12} c_{23}\right)$ with projection $\bar{\alpha}$ to its homogeneous parts with degree in $Y$. If $x, y, z \in \operatorname{supp} V$ and two of

$$
\left\{(x, y, z), \sigma_{2} \cdot(x, y, z), \sigma_{1} \sigma_{2} \cdot(x, y, z)\right\}
$$

are in $Y$, then for any $\alpha \in k(Y, \bar{\alpha})$ the summand with the third degree is uniquely determined. Thus $k(Y, \bar{\alpha}) \subseteq k\left(Y^{\prime}, \bar{\alpha}^{\prime}\right)$, where $Y^{\prime}$ is the union of $Y$ and the third degree and $\bar{\alpha}^{\prime}$ is the extension of $\bar{\alpha}$. This procedure of enlarging $Y$ can be regarded as a cellular automaton on $(\operatorname{supp} V)^{3}$. If $Y=(\operatorname{supp} V)^{3}$, then $k(Y, \bar{\alpha})=\bar{\alpha}$ or $k(Y, \bar{\alpha})=\emptyset$. Hence, if a given subset $Y \subseteq(\operatorname{supp} V)^{3}$ can be enlarged this way to $(\operatorname{supp} V)^{3}$, then the projection of $\operatorname{ker}\left(1+c_{12}+c_{12} c_{23}\right)$ to the sum of homogeneous parts of degree $(x, y, z) \in Y$ is injective. Thus an important question is the following: for a given Hurwitz orbit $\mathcal{O}$, provide (the size of) a smallest subset $Y$ which can be enlarged by the above process to $\mathcal{O}$. The size of such a $Y$ yields surprisingly often a sharp upper bound for $\operatorname{dim} \operatorname{ker}\left(1+c_{12}+c_{12} c_{23}\right)$. We call the quotient $|Y| /|\mathcal{O}|$ the immunity of $\mathcal{O}$.

Our classification of finite-dimensional elementary Nichols algebras with many cubic relations has three steps. First we solve the evolution problem on the Hurwitz orbits in $X^{3}$ for all indecomposable racks $X$ such that the quotient by the center has only $\sigma_{1}$-cycles of length $\leq 4$. This is one of our main results. Then we conclude a small upper bound for the size of possible racks. Using additional information on the cycle structure of racks and the classification of indecomposable racks of size $\leq 35$, we finally obtain all racks satisfying the inequality on the immunity of $X^{3}$. For those racks we then study the possible cocycles coming from Yetter-Drinfeld structures of the enveloping group $G_{X}$ and find all elementary Nichols algebras with many cubic relations under the standing assumption on the Hurwitz orbit structure. This finishes our classification.

The paper is organized as follows. In Section 2 we review the basic definitions concerning racks and we prove our first main result about the structure of indecomposable racks. In Section 3 we provide an obstruction on the cycle structure of indecomposable crossed sets. Section 4 is devoted to the theory of coverings of Hurwitz orbits. In Section 5 we define cellular automata on $G$-spaces for arbitrary groups $G$ and study particular examples. In Section 7 we prove our second main result formulated in Section 6 an explicit upper bound for the immunity of a class 
of Hurwitz orbits in terms of local data. Finally, in Sections 8 and 9 we classify elementary Nichols algebras with many cubic relations under our standing assumption on Hurwitz orbits.

\section{RACKS}

We recall basic notions and facts about racks. For additional information we refer to [1]. A rack is a pair $(X, \triangleright)$, where $X$ is a non-empty set and $\triangleright: X \times X \rightarrow X$ is a map (considered as a binary operation on $X$ ) such that

(1) the map $\varphi_{i}: X \rightarrow X$, where $x \mapsto i \triangleright x$, is bijective for all $i \in X$, and

(2) $i \triangleright(j \triangleright k)=(i \triangleright j) \triangleright(i \triangleright k)$ for all $i, j, k \in X$ (i. e. $\triangleright$ is self-distributive).

A rack $(X, \triangleright)$, or for short $X$, is a quandle if $i \triangleright i=i$ for all $i \in X$. A crossed set $X$ is a quandle such that $x \triangleright y=y$ if and only if $y \triangleright x=x$ for all $x, y \in X$. A subrack of a rack $X$ is a non-empty subset $Y \subseteq X$ such that $(Y, \triangleright)$ is also a rack. The inner group of a rack $X$ is the group generated by the permutations $\varphi_{i}$ of $X$, where $i \in X$. We write $\operatorname{Inn}(X)$ for the inner group of $X$. For any rack $X$, the enveloping group of $X$ is the group $G_{X}$ given by the generating set $X$ and the relations $x y=(x \triangleright y) x$ for all $x, y \in X$. A rack $X$ is called injective if the canonical map $X \rightarrow G_{X}$ is injective.

We say that a rack $X$ is indecomposable if the inner group $\operatorname{Inn}(X)$ acts transitively on $X$. Also, $X$ is decomposable if it is not indecomposable. The profile of an indecomposable rack $X$ is the cycle structure of the permutation $\varphi_{x}$ for some $x \in X$. For example, a profile $1^{a} 2^{b} 3^{c}$ means that for any $x \in X, \varphi_{x}$ is a product of $b$ disjoint transpositions, $c$ disjoint 3-cycles and $|X|=a+b+c$.

Example 2.1. A group $G$ is a rack with $x \triangleright y=x y x^{-1}$ for all $x, y \in G$. If a subset $X \subseteq G$ is stable under conjugation by the elements of $X$, then it is a subrack of $G$. In particular, the conjugacy class $g^{G}$ of any $g \in G$ is a rack.

Remark 2.2. For a union $X$ of conjugacy classes of a group $G$, the subgroup of $G$ generated by $X$ is a quotient of the enveloping group of $X$. This implies that a rack is injective if and only if it is isomorphic to a union of conjugacy classes of a group.

Example 2.3. Let $A$ be an abelian group, and $X=A$. For any $g \in \operatorname{Aut}(A)$ we have a rack structure on $X$ given by

$$
x \triangleright y=(1-g) x+g y
$$

for all $x, y \in X$. This rack is called the affine rack associated to the pair $(A, g)$ and will be denoted by $\operatorname{Aff}(A, g)$. In particular, let $p$ be a prime number, $q$ a power of $p$ and $\alpha \in \mathbb{F}_{q} \backslash\{0,1\}$. We write $\operatorname{Aff}\left(\mathbb{F}_{q}, \alpha\right)$, or simply $\operatorname{Aff}(q, \alpha)$, for the affine rack $\operatorname{Aff}(A, g)$, where $A=\mathbb{F}_{q}$ and $g$ is the automorphism given by $x \mapsto \alpha x$ for all $x \in \mathbb{F}_{q}$.

Example 2.4. Let $p$ be a prime number. The affine rack $\operatorname{Aff}(p,-1)$ is called a dihedral rack and will be denoted by $\mathbb{D}_{p}$. 
Let $X$ be a finite indecomposable injective rack and let $x \in X$. In [5, Section 2.3 integers $k_{n}$ for $n \in \mathbb{N}_{\geq 2}$ were defined by

$$
\begin{aligned}
& k_{n}=\mid\{y \in X \mid \underbrace{x \triangleright(y \triangleright(x \triangleright(y \triangleright \cdots)))}_{n \text { elements }}=y, \\
&\underbrace{x \triangleright(y \triangleright(x \triangleright(y \triangleright \cdots)))}_{j \text { elements }} \neq y \text { for all } j \in\{1,2, \ldots, n-1\}\} \mid .
\end{aligned}
$$

Since $X$ is indecomposable, the numbers $k_{n}$ do not depend on $x$. Recall that for all $x \in X$ the permutation $\varphi_{x}$ has precisely $1+k_{2}$ fixed points. Furthermore, $1+k_{2}+k_{3}+\cdots$ is just the cardinality of $X$. We frequently use the notation

$$
k_{m}^{\prime}=k_{m+1}+k_{m+2}+k_{m+3}+\cdots
$$

where $m \in \mathbb{N}$. The number of points moved by $\varphi_{x}$ is then $k_{2}^{\prime}=k_{3}+k_{3}^{\prime}$.

Lemma 2.5. Let $X$ be a crossed set and let $u \in X$. Then the subset $S=$ $\{x \in X \mid u \triangleright x=x, x \neq u\}$ is a subquandle of $X$.

Proof. Let $x, y \in X$ with $u \triangleright x=x, u \triangleright y=y$. Then

$$
u \triangleright(x \triangleright y)=(u \triangleright x) \triangleright(u \triangleright y)=x \triangleright y .
$$

Further, $x \triangleright u=u$ since $X$ is a crossed set and hence $\varphi_{x}(S) \subseteq S$. Clearly, $\left.\varphi_{x}\right|_{S}$ is injective. Finally, $\varphi_{x}^{-1}(S \cup\{u\}) \subseteq S \cup\{u\}$ since

$$
u \triangleright\left(\varphi_{x}^{-1}(y)\right)=\varphi_{u \triangleright x}^{-1}(u \triangleright y)=\varphi_{x}^{-1}(y)
$$

and $\varphi_{x}^{-1}(u)=u$. Thus $\left.\varphi_{x}\right|_{S}$ is invertible with inverse $\left.\varphi_{x}^{-1}\right|_{S}$.

Proposition 2.6. Let $X$ be an indecomposable crossed set and let $u \in X$. Let $T=\{x \in X \mid u \triangleright x \neq x\}$. Then for all $t \in T$ there exists $t^{\prime} \in T$ with $t \triangleright t^{\prime} \neq t^{\prime}$.

Proof. Let $S=X \backslash(T \cup\{u\})$ and

$$
T_{0}=\left\{t \in T \mid \operatorname{supp} \varphi_{t} \subseteq S \cup\{u\}\right\} .
$$

We have to show that $T_{0}=\emptyset$.

Assume that $T_{0} \neq \emptyset$. Since $X$ is indecomposable, there exist $x \in T_{0}, y \in X \backslash T_{0}$, $z \in X$ such that $z \triangleright x=y$. As $x \triangleright z \neq z$ and $x \in T_{0}$, we conclude that $z \in S \cup\{u\}$. As $S \cup\{u\}$ is a subquandle of $X$ by Lemma 2.5 and $x=\varphi_{z}^{-1}(y)$, it follows that $y \in T \backslash T_{0}$. But $\operatorname{supp} \varphi_{z \triangleright x}=\varphi_{z}\left(\operatorname{supp} \varphi_{x}\right) \subseteq S \cup\{u\}$, a contradiction to $z \triangleright x=y \in$ $T \backslash T_{0}$. This proves the proposition.

Theorem 2.7. Let $X$ be an indecomposable crossed set. Assume that the profile of $X$ is $1^{a_{1}} 2^{a_{2}} \ldots k^{a_{k}}$. Then

$$
k_{2} \leq \sum_{j \geq 2} a_{j}\left(k_{2}^{\prime}-2\right) \quad \text { and } \quad|X| \leq \sum_{j \geq 2} a_{j}\left(k_{2}^{\prime}-2\right)+k_{2}^{\prime}+1 .
$$

Proof. Let $u \in X, T=\{x \in X \mid u \triangleright x \neq x\}$ and $S=X \backslash(T \cup\{u\})$. Then $S$ is a subquandle of $X$ by Lemma 2.5. Let $S_{1}, \ldots, S_{n}$ be the $\operatorname{Inn}(S)$ orbits of $S$. Then $S \triangleright S_{j}=S_{j}$ for all $j$, and $S \triangleright T=T$ because $S$ is a subquandle and $S \triangleright\{u\}=\{u\}$. Since $X$ is indecomposable, there can only be elements of $T$ to connect the $\operatorname{Inn}(S)$ orbits $S_{j}$ with each other, with $\{u\}$ and with $T$.

Let $x \in T, s \in S_{j}$ and $y \in X \backslash S_{j}$ be such that $x \triangleright y=s . \operatorname{Applying} \operatorname{Inn}(S)$ one can see that for all $s^{\prime} \in S_{j}$ there must be elements $x^{\prime} \in T, y^{\prime} \in X \backslash S_{j}$ with $x^{\prime} \triangleright y^{\prime}=s^{\prime}$. Hence, if one element of $S_{j}$ is connected to $X \backslash S_{j}$ via a single element 
of $T$, then every element of $S_{j}$ is. We conclude that each of the $k_{2}$ elements of $S$ has to be moved by at least one $\varphi_{x}$ for an $x \in T$.

Each $\varphi_{x}, x \in T$, moves $k_{2}^{\prime}$ elements within $X$. One of these elements must be $u$, and by Proposition 2.6 another one must be an element of $T$. So there are at most $k_{2}^{\prime}-2$ elements which may be moved by $\varphi_{x}$ from $S$ to $X$ for each $x \in T$, and $k_{2}^{\prime}\left(k_{2}^{\prime}-2\right)$ altogether. Let

$$
Y=\{(x, s) \mid x \in T, s \in S, x \triangleright s \neq s\} .
$$

For any $(x, s) \in Y, \varphi_{u}(x) \triangleright s=\varphi_{u}(x) \triangleright \varphi_{u}(s)=\varphi_{u}(x \triangleright s) \neq \varphi_{u}(s)=s$, and $\varphi_{u}(x) \neq x$. So each $s \in S$ appears not only once as a second component in some $(x, s) \in Y$, but at least $j$ times, where $j=\min \left\{m \in \mathbb{N} \mid \varphi_{u}^{m}(x)=x\right\}$. For each $j$, there are $j a_{j}$ such $x \in T$, which provide ( since $x \triangleright u \neq u$ ) at most $j a_{j}\left(k_{2}^{\prime}-2\right)$ pairs $(x, s) \in Y$. In these pairs we may have up to $a_{j}\left(k_{2}^{\prime}-2\right)$ different elements from $S$ as a second component, and hence $k_{2} \leq \sum_{j \geq 2} a_{j}\left(k_{2}^{\prime}-2\right)$. This proves the first formula of the claim. The second now follows immediately from $|X|=1+k_{2}+k_{2}^{\prime}$.

Corollary 2.8. Under the assumptions of Theorem 2.7, $k_{2} \leq \frac{1}{2} k_{2}^{\prime}\left(k_{2}^{\prime}-2\right)$ and $|X| \leq \frac{1}{2} k_{2}^{\prime 2}+1$.

Proof. Use the inequality $\sum_{j \geq 2} a_{j} \leq \frac{1}{2} \sum_{j \geq 2} j a_{j}=k_{2}^{\prime} / 2$.

\section{ON THE CYCLE STRUCTURE OF RACKS}

Lemma 3.1. Let $d \in \mathbb{N}, a, b \in \mathbb{N}_{\geq 2}$ with $\operatorname{gcd}(a, b)=1$ and let $X=\{1, \ldots, d\}$ be an indecomposable crossed set. Assume that $\varphi_{1}^{a}(x)=x$ or $\varphi_{1}^{b}(x)=x$ for all $x \in X$. Let

$$
\begin{aligned}
S & =\{x \in X \mid 1 \triangleright x=x, x \neq 1\}, \\
T_{a} & =\left\{x \in X \mid \varphi_{1}^{a}(x)=x, 1 \triangleright x \neq x\right\}, \\
T_{b} & =\left\{x \in X \mid \varphi_{1}^{b}(x)=x, 1 \triangleright x \neq x\right\} .
\end{aligned}
$$

Then $X$ is the disjoint union of $\{1\}, S, T_{a}$ and $T_{b}$ and we have

$$
\begin{aligned}
& 1 \triangleright 1=1, \quad 1 \triangleright S=S, \quad 1 \triangleright T_{a}=T_{a}, \quad 1 \triangleright T_{b}=T_{b}, \\
& S \triangleright 1=1, \quad S \triangleright S=S, \quad S \triangleright T_{a}=T_{a}, \quad S \triangleright T_{b}=T_{b}, \\
& T_{a} \triangleright T_{b}=T_{b}, \quad T_{b} \triangleright T_{a}=T_{a} .
\end{aligned}
$$

Proof. The action of 1 is clear by the definition of $S, T_{a}$ and $T_{b} . X$ is a crossed set; therefore $s \triangleright 1=1$ holds for each $s \in S$. Moreover, $S \triangleright S=S$ by Lemma 2.5. Let $s \in S$ and $t \in T_{a}$ be arbitrary. Then $s \triangleright t=s \triangleright \varphi_{1}^{a}(t)=\varphi_{1}^{a}(s \triangleright t)$ because 1 and $s$ commute. Hence, $s \triangleright t \in T_{a} \cup S \cup\{1\}$. But $\varphi_{s}$ is bijective, so $s \triangleright t \in T_{a}$; similarly for $t \in T_{b}$. Now let $x \in T_{a}$ and $y \in T_{b}$ be arbitrary. Assume $x \triangleright y \in T_{a} \cup S \cup\{1\}$. Then apply $\varphi_{1}^{a}$ to get $x \triangleright \varphi_{1}^{a}(y)=x \triangleright y$; hence $\varphi_{1}^{a}(y)=y$, which is a contradiction. So $T_{a} \triangleright T_{b}=T_{b}$ and similarly $T_{b} \triangleright T_{a}=T_{a}$.

Proposition 3.2. Let $r, d \in \mathbb{N}_{\geq 1}, a_{1}, \ldots, a_{r}, b \in \mathbb{N}_{\geq 2}$ with $\operatorname{gcd}\left(a_{1} \cdots a_{r}, b\right)=1$ and $d \geq a_{1}+\cdots+a_{r}+b+1$. For all $i \in\{1, \ldots, r\}$ let $\sigma_{i}$ be an $a_{i}$-cycle and let $\tau$ be a b-cycle in $\mathbb{S}_{d}$ with pairwise disjoint supports. Then there is no indecomposable crossed set $X=\{1, \ldots, d\}$ with $\varphi_{1}=\sigma_{1} \cdots \sigma_{r} \tau$. 
Proof. Assume to the contrary that $X$ is an indecomposable crossed set with $\varphi_{1}=$ $\sigma_{1} \cdots \sigma_{r} \tau$. Then $1 \notin \operatorname{supp} \sigma_{i}$ for all $i$ and $1 \notin \operatorname{supp} \tau$. Let $y_{1}, \ldots, y_{b} \in X$ with $1 \triangleright y_{i}=y_{i+1}$ for all $1 \leq i<b$ and $1 \triangleright y_{b}=y_{1}$. Then

$$
\tau=\left(y_{1} \cdots y_{b}\right) \text {. }
$$

Let

$$
T=\bigcup_{i=1}^{r} \operatorname{supp} \sigma_{i}, \quad S=X \backslash(\{1\} \cup T \cup \operatorname{supp} \tau) .
$$

Then Lemma 3.1 applies with $a=a_{1} \cdots a_{r}, T_{a}=T, T_{b}=\operatorname{supp} \tau$. We consider four cases.

Case 1. $y_{1} \triangleright 1 \in S$. Let $s=y_{1} \triangleright 1$. Then

$$
\varphi_{s}=\varphi_{y_{1} \triangleright 1}=\varphi_{y_{1}} \triangleright \varphi_{1}=\varphi_{y_{1}} \sigma_{1} \cdots \sigma_{r} \varphi_{y_{1}}^{-1} \cdot\left(y_{1} y_{1} \triangleright y_{2} \cdots y_{1} \triangleright y_{b}\right) \text {. }
$$

As $s \in S$, we know from Lemma 3.1 that $s \triangleright \operatorname{supp} \tau=\operatorname{supp} \tau$ and hence $y_{1} \in \operatorname{supp} \tau$ implies that $y_{1} \triangleright y_{i} \in \operatorname{supp} \tau$ for all $i \in\{1, \ldots, b\}$. Thus $y_{1} \triangleright \operatorname{supp} \tau=\operatorname{supp} \tau$. Applying $\varphi_{1}$ and using the transitivity of the action of $\varphi_{1}$ on $\operatorname{supp} \tau$ we conclude that $y_{i} \triangleright \operatorname{supp} \tau=\operatorname{supp} \tau$ for all $i \in\{1, \ldots, b\}$. Thus $\operatorname{supp} \tau$ is $X$-stable by Lemma 3.1. which contradicts the indecomposability of $X$.

Case 2. $y_{1} \triangleright 1 \in T$. Since $y_{1} \in \operatorname{supp} \tau$, Lemma 3.1 yields that $y_{1} \triangleright T=T$ and hence $\varphi_{y_{1}}^{-1}(T)=T$, a contradiction to $1 \in \varphi_{y_{1}}^{-1}(T)$.

Case 3. $y_{1} \triangleright 1 \in \operatorname{supp} \tau, \varphi_{y_{1}}^{a_{1} \cdots a_{r}}(1)=1$. Let $a=a_{1} \cdots a_{r}$. Then $\varphi_{y_{1}}^{a} \varphi_{1} \varphi_{y_{1}}^{-a}=\varphi_{1}$, and hence $\varphi_{y_{1}}^{a} \tau \varphi_{y_{1}}^{-a}=\tau$. Since $\varphi_{y_{1}}\left(y_{1}\right)=y_{1}$, we conclude that $\varphi_{y_{1}}^{a}(y)=y$ for all $y \in \operatorname{supp} \tau$. Conjugation by $\varphi_{1}$ yields

$$
\varphi_{y}^{a}\left(y^{\prime}\right)=y^{\prime} \quad \text { for all } y, y^{\prime} \in \operatorname{supp} \tau .
$$

By assumption, $y_{1} \triangleright 1 \in \operatorname{supp} \tau$. Further,

$$
\varphi_{y_{1} \triangleright 1}=\varphi_{y_{1}} \varphi_{1} \varphi_{y_{1}}^{-1}=\varphi_{y_{1}} \sigma_{1} \cdots \sigma_{r} \varphi_{y_{1}}^{-1} \cdot\left(y_{1} y_{1} \triangleright y_{2} \cdots y_{1} \triangleright y_{b}\right) .
$$

Since $b \nmid a$, we conclude that $\varphi_{y_{1} \triangleright 1}^{a}\left(y_{1}\right) \neq y_{1}$, which is a contradiction to equation (3.1).

Case 4. $y_{1} \triangleright 1 \in \operatorname{supp} \tau, \varphi_{y_{1}}^{b}(1)=1$. By applying $\varphi_{1}$ and using Lemma 3.1 we obtain that $y \triangleright 1 \in \operatorname{supp} \tau$ and $\varphi_{y}^{b}(1)=1$ for all $y \in \operatorname{supp} \tau$. In particular, for all $y \in \operatorname{supp} \tau$ there exist $\sigma_{y, 1}, \ldots, \sigma_{y, r}, \tau_{y} \in \mathbb{S}_{d}$ with $1 \in \operatorname{supp} \tau_{y}$ such that $\varphi_{y}=\sigma_{y, 1} \cdots \sigma_{y, r} \tau_{y}$. Since

$$
\varphi_{y_{1} \triangleright 1}=\prod_{i=1}^{r}\left(\varphi_{y_{1}} \sigma_{i} \varphi_{y_{1}}^{-1}\right) \cdot\left(y_{1} y_{1} \triangleright y_{2} \cdots y_{1} \triangleright y_{b}\right)
$$

and $y_{1} \triangleright T=T$ by Lemma 3.1, we conclude that $\operatorname{supp} \sigma_{y_{1} \triangleright 1, i} \subseteq T$ for all $i \in$ $\{1, \ldots, r\}$. By conjugation of equation (3.2) with $\varphi_{1}$ and using $1 \triangleright T=T$ and the transitivity of $\varphi_{1}$ on $\operatorname{supp} \tau$ it follows that

$$
\operatorname{supp} \sigma_{y, 1} \cdots \sigma_{y, r}=T \quad \text { for all } y \in \operatorname{supp} \tau .
$$

Let $x \in T, y \in \operatorname{supp} \tau, z \in X$ with $x \triangleright y=z$. Then $z \in \operatorname{supp} \tau$ by Lemma 3.1 . Further,

$$
\sigma_{z, 1} \cdots \sigma_{z, r} \tau_{z}=\varphi_{z}=\varphi_{x \triangleright y}=\varphi_{x}\left(\sigma_{y, 1} \cdots \sigma_{y, r}\right) \varphi_{x}^{-1} \cdot \varphi_{x} \tau_{y} \varphi_{x}^{-1}
$$


and hence $\sigma_{z, 1} \cdots \sigma_{z, r}=\varphi_{x}\left(\sigma_{y, 1} \cdots \sigma_{y, r}\right) \varphi_{x}^{-1}$. Thus $\varphi_{x}(T)=T$. We conclude that $T \triangleright T=T$ and hence $X \triangleright T=T$ by Lemma 3.1, a contradiction to the indecomposability of $X$.

\section{Quotients of Hurwitz orbits}

For the general theory of braid groups we refer to [8]. Recall that the braid group in three strands can be presented as

$$
\mathbb{B}_{3}=\left\langle\sigma_{1}, \sigma_{2} \mid \sigma_{1} \sigma_{2} \sigma_{1}=\sigma_{2} \sigma_{1} \sigma_{2}\right\rangle .
$$

Let $X$ be a finite rack and $(x, y, z) \in X^{3}$. Then the braid group $\mathbb{B}_{3}$ acts on $X^{3}$ via the Hurwitz action:

$$
\sigma_{1} \cdot(x, y, z)=(x \triangleright y, x, z), \quad \sigma_{2} \cdot(x, y, z)=(x, y \triangleright z, y)
$$

for all $x, y, z \in X$. The orbits of this action are called Hurwitz orbits. We write $\mathcal{O}=\mathcal{O}(x, y, z)$ for the Hurwitz orbit of $(x, y, z)$. According to Brieskorn [3], this action of $\mathbb{B}_{3}$ on $X^{3}$ was implicitly considered by Hurwitz in [7].

Lemma 4.1. Let $X$ be a rack and $\mathcal{O} \subseteq X^{3}$ a Hurwitz orbit. Then the map $\mathcal{O} \rightarrow$ $G_{X},(x, y, z) \mapsto x y z$ is constant.

Proof. According to the definition of the Hurwitz action, it suffices to show that $x y=(x \triangleright y) x$ for all $x, y \in X$. The latter holds by the definition of $G_{X}$.

Lemma 4.2. Let $X$ be an injective rack, $\mathcal{O} \subseteq X^{3}$ a Hurwitz orbit and $(x, y, z) \in \mathcal{O}$. Then $\left|(x, y, z)^{\left\langle\sigma_{1}\right\rangle} \cap(x, y, z)^{\left\langle\sigma_{2}\right\rangle}\right|=1$.

Proof. The elements of $(x, y, z)^{\left\langle\sigma_{1}\right\rangle} \cap(x, y, z)^{\left\langle\sigma_{2}\right\rangle}$ are of the form $\left(x, y^{\prime}, z\right)$ with $y^{\prime} \in$ $X$. For all such triples, $x y z=x y^{\prime} z$ in $G_{X}$ by Lemma 4.1. Thus $y=y^{\prime}$ in $G_{X}$ and hence $y=y^{\prime}$ in $X$ since $X$ is injective.

Let $(x, y, z),\left(x^{\prime}, y^{\prime}, z^{\prime}\right) \in X^{3}$. We define the following relation on $X^{3}$ :

$$
(x, y, z) \sim\left(x^{\prime}, y^{\prime}, z^{\prime}\right) \Leftrightarrow \Delta^{m} \cdot(x, y, z)=\left(x^{\prime}, y^{\prime}, z^{\prime}\right) \text { for some } m \in \mathbb{Z},
$$

where $\Delta=\left(\sigma_{1} \sigma_{2}\right)^{3}$. Clearly, $\sim$ is an equivalence relation. We write $\overline{\mathcal{O}}(x, y, z)$ for the equivalence class containing $(x, y, z)$. The set $\overline{\mathcal{O}}$ of equivalence classes of $\mathcal{O}$ is called a Hurwitz orbit quotient.

Let $x=\sigma_{2}^{-1} \sigma_{1}^{-1} Z\left(\mathbb{B}_{3}\right)$ and $y=\sigma_{1} \sigma_{2} \sigma_{1} Z\left(\mathbb{B}_{3}\right)$. Then, by construction, the group

$$
\left\langle x, y \mid x^{3}=y^{2}=1\right\rangle \simeq \operatorname{PSL}(2, \mathbb{Z}) \simeq \mathbb{B}_{3} /\langle\Delta\rangle
$$

acts on $\overline{\mathcal{O}}$; see for example [8, Appendix A]. Since $\mathbb{B}_{3}$ acts transitively on $\mathcal{O}$, the action of $\operatorname{PSL}(2, \mathbb{Z})$ on $\overline{\mathcal{O}}$ is transitive; that is, $\overline{\mathcal{O}}$ is a homogeneous space.

An $x y$-cycle in a $\mathbf{P S L}(2, \mathbb{Z})$-space (in particular, in a Hurwitz orbit quotient) is a minimal non-empty subset $C$ such that $x y \cdot v \in C$ and $(x y)^{-1} \cdot v \in C$ for all $v \in C$. We write $C_{x y}(v)$ for the $x y$-cycle containing a fixed element $v$. Similarly, one defines $y x$-cycles $C_{y x}(v)$.

We intend to determine all finite homogeneous $\operatorname{PSL}(2, \mathbb{Z})$-spaces (up to isomorphism) such that any $x y$-cycle has at most four elements. Finite homogeneous $\operatorname{PSL}(2, \mathbb{Z})$-spaces up to isomorphism are known to be in bijection with conjugacy classes of finite index subgroups of the modular group $\mathbf{P S L}(2, \mathbb{Z})$, which are intensively studied; see e.g. 9]. We present such sets in terms of their Schreier graphs with respect to the generators $x$ and $y$ of $\mathbf{P S L}(2, \mathbb{Z})$. Here a Schreier graph of 
a subgroup $H \subseteq \mathbf{P S L}(2, \mathbb{Z})$ will be an oriented labeled graph with vertices corresponding to the left $H$-cosets. In the interpretation as a $\mathbf{P S L}(2, \mathbb{Z})$-space, the vertices correspond to the points of the space. In the Schreier graph, an $x$-arrow points from any coset $g H$ (equivalently, point of the $\mathbf{P S L}(2, \mathbb{Z}$ )-space) to the coset $x g H$, and a $y$-arrow points from any coset $g H$ to the coset $y g H$. Instead of a double arrow labeled by $y$ we display a single dashed line. We then omit the label $x$ on the $x$-arrows. Later on we will add further labels on the graph which is used to study Schreier graphs of finite index subgroups of coverings of $\mathbf{P S L}(2, \mathbb{Z})$.

Proposition 4.3. Let $M$ be a finite homogeneous $\mathbf{P S L}(2, \mathbb{Z})$-space such that any $x y$-cycle in $M$ has at most four elements. Then the Schreier graph of $M$ is one of the graphs in Figures 7.17 .18 .

We denote the homogeneous $\mathbf{P S L}(2, \mathbb{Z})$-spaces and their Schreier graphs appearing in Proposition 4.3 by $\Sigma_{n X}$, where $n$ denotes the number of vertices of the graph and $X$ is a capital letter which serves as a further distinction.

Proof. The calculations are somewhat lengthy but elementary. It is reasonable to start with the classification of all homogeneous spaces with a point fixed by $x$, that is, with Schreier graphs containing an oriented loop. The restriction on the cycles yields that only the Schreier graphs in Figures 7.1, 7.2, 7.5, 7.6, 7.11, and 7.12 are possible. We explain this step in detail.

Let $v_{1}$ be a point of $M$ with $x v_{1}=v_{1}$. If $y v_{1}=v_{1}$, then the Schreier graph of $M$ is in Figure [7.1. Otherwise, let $v_{2}=y v_{1}$. If $x v_{2}=v_{2}$, then the Schreier graph of $M$ is in Figure 7.2. Assume now that $x v_{2} \neq v_{2}$. Since $x^{3} v_{2}=v_{2}$, we have at least two more points $v_{3}=x v_{2}$ and $v_{4}=x v_{3}$, and then $x v_{4}=v_{2}$. Recall that each vertex of the Schreier graph has precisely one incoming and one outgoing black arrow and one outgoing dashed line, which implies that $v_{3}, v_{4}$ can neither be equal to an existing point nor to each other. If $y v_{3}=v_{4}$, then the Schreier graph of $M$ is in Figure [7.5. If $y v_{4}=v_{4}$, then let $v=y v_{3}$. The $x y$-cycle through $v$ contains the vertices $v, x y v=v_{4}, x y v_{4}=v_{2}, x y v_{2}=v_{1}$, and $x y v_{1}=v_{3}$. Since it can contain at most four vertices, we conclude that $v=v_{3}$. Similarly, $y v_{3}=v_{3}$ implies that $y v_{4}=v_{4}$. Then the Schreier graph of $M$ is in Figure 7.6. Assume now that $y v_{3} \neq v_{3}$ and $y v_{4} \neq v_{4}$. Then we have at least two more points, $v_{5}=y v_{3}$ and $v_{6}=y v_{4}$. Since $(x y)^{m} v_{6}=v_{6}$ for some $m \in\{1,2,3,4\}$ and since $x y v_{6}=v_{2}$, $(x y)^{2} v_{6}=v_{1},(x y)^{3} v_{6}=v_{3}$, we obtain that $v_{6}=x y v_{3}=x v_{5}$. Since $x^{3} v_{5}=v_{5}$, there is a point $v_{7}=x v_{6}$ of $M$, and $x v_{7}=v_{5}$. If $y v_{7}=v_{7}$, then the Schreier graph of $M$ is in Figure 7.11. Finally, let $v_{8}=y v_{7}$. Then $v_{8}, v_{5}=x y v_{8}, v_{4}=x y v_{5}$, and $v_{7}=x y v_{4}$ are pairwise distinct, and hence $x y v_{7}=v_{8}$ by assumption on the length of $x y$-cycles. Thus $x v_{8}=v_{8}$. The Schreier graph of $M$ is then in Figure 7.12

Next, one proceeds with the homogeneous spaces having a point fixed by $x y$ but no point fixed by $x$. The corresponding Schreier graphs are Figures 7.3 and 7.7 Next, one can determine those homogeneous spaces which have a point fixed by $y$, but no points fixed by $x$ or $x y$. The corresponding Schreier graphs are Figures 7.4. 7.8, 7.9, 7.13, and 7.14, Finally, only Schreier graphs are left which have no oriented loops and no $y$-edges starting and ending in the same oriented triangle. Such graphs can be classified for example by looking at the number of $y$-edges between two oriented triangles. Under the restriction on the length of $x y$-cycles, one gets the Figures 7.10, 7.15, 7.16, 7.17, and 7.18, 
For our analysis, finite homogeneous $\mathbb{B}_{3}$-spaces are relevant. We obtain such spaces as coverings of homogeneous $\operatorname{PSL}(2, \mathbb{Z})$-spaces.

Definition 4.4. Let $T$ be a $\operatorname{PSL}(2, \mathbb{Z})$-space. Consider $T$ as a $\mathbb{B}_{3}$-space on which $Z\left(\mathbb{B}_{3}\right)$ acts trivially. A covering of $T$ is a triple $(p, S, T)$, where $S$ is a $\mathbb{B}_{3}$-space, and $p: S \rightarrow T$ is a surjective $\mathbb{B}_{3}$-equivariant map such that $p\left(s_{1}\right)=p\left(s_{2}\right)$ for $s_{1}, s_{2} \in S$ implies that $s_{1}=\Delta^{m} s_{2}$ for some $m \in \mathbb{Z}$.

Remark 4.5. Let $T$ be a $\mathbf{P S L}(2, \mathbb{Z})$-space and let $(p, S, T)$ be a covering of $T$. If the action on $T$ is transitive, then the action on $S$ is transitive.

Definition 4.6. A covering $(p, S, T)$ is said to be trivial if $p: S \rightarrow T$ is bijective. We say that a covering $p: S \rightarrow T$ is finite if $S$ (and hence $T$ ) is a finite set.

Since $\Delta$ is contained in (and in fact generates) the center $Z\left(\mathbb{B}_{3}\right)$, the following lemma is straightforward.

Lemma 4.7. Let $(p, S, T)$ be a covering of a homogeneous $\operatorname{PSL}(2, \mathbb{Z})$-space $T$. Then $\left|p^{-1}(v)\right|=\left|p^{-1}(w)\right|$ for all points $v, w$ of $T$.

Let $(p, S, T)$ be a covering of a homogeneous $\mathbf{P S L}(2, \mathbb{Z})$-space $T$. We will always write $N$ for the number of elements of the fibers.

Remark 4.8. Let $T$ be a homogeneous $\operatorname{PSL}(2, \mathbb{Z})$-space. Coverings $(p, S, T)$ of $T$ can be displayed as labeled Schreier graphs with respect to the generators $\sigma_{2}^{-1} \sigma_{1}^{-1}$ and $\sigma_{1} \sigma_{2} \sigma_{1}$ of $\mathbb{B}_{3}$. Hence, by definition of $x$ and $y$, the generators $\sigma_{2}^{-1} \sigma_{1}^{-1}$ and $\sigma_{1} \sigma_{2} \sigma_{1}$ correspond to labeled $x$ - and $y$-arrows, respectively, in the labeled Schreier graph. Since the covering is a homogeneous space and the sequence

$$
Z\left(\mathbb{B}_{3}\right) \rightarrow \mathbb{B}_{3} \rightarrow \mathbf{P S L}(2, \mathbb{Z})
$$

is exact, the fiber over any $v \in T$ consists of a $\langle\Delta\rangle$-orbit. We may fix a point $v[0] \in S$ in the fiber over a point $v \in T$ and enumerate all other points of the fiber by $v[i]=\Delta^{i} v[0]$ for all $i \in\{0,1, \ldots, N-1\}$, where $N$ is the size of the fiber. We also write $v[*]$ for the complete fiber $p^{-1}(v)$ over $v$. By choosing a spanning tree of the Schreier graph of $T$ and the images of $v[0]$ along the arrows of the spanning tree, one obtains the images of $v[i]$ for all $i$ since $\Delta$ is central. The remaining arrows $v_{i} \rightarrow v_{j}$ (those not on the spanning tree) in the graph of $T$ then have to obtain labels indicating the index shift in the fiber: a label $s$ tells that $v_{i}[k]$ is mapped to $v_{j}[k+s(\bmod N)]$ for all $k$. Then, up to the choice of the spanning tree, any covering of $T$ is uniquely determined by the labels of the $x$ - and $y$-edges.

Observe that since $\Delta=\left(\sigma_{1} \sigma_{2}\right)^{3}=\left(\sigma_{1} \sigma_{2} \sigma_{1}\right)^{2}$, the sum of the labels in any $x$ triangle is -1 and the sum of the two labels of a $y$-edge is 1 . The $y$-edges we interpret as double arrows and put the label of the arrow close to its destination.

For any $x y$-cycle (or $y x$-cycle) $C$ in $T$, the label of $C$ is the sum of the labels of $x$ - and $y$-edges of the cycle.

Definition 4.9. Let $\mathcal{O}$ be a covering of a $\operatorname{PSL}(2, \mathbb{Z})$-space. The graph of the covering is the labeled Schreier graph as explained in Remark 4.8. The arrows correspond to the action of $\sigma_{2}^{-1} \sigma_{1}^{-1}$ and $\sigma_{1} \sigma_{2} \sigma_{1}$.

Definition 4.10. For $i \in\{1,2\}$, a $\sigma_{i^{-}}$cycle of a homogeneous $\mathbb{B}_{3}$-space is a minimal non-empty subset which is closed under the action of $\sigma_{i}$. We say that a $\mathbb{B}_{3}$-space $S$ (or a covering $(p, S, T)$ of a $\mathbf{P S L}(2, \mathbb{Z})$-space $T$ ) has simply intersecting cycles if any given $\sigma_{1}$-cycle $c_{1}$ and $\sigma_{2}$-cycle $c_{2}$ in $S$ intersect in at most one point. 
Example 4.11. By Lemma 4.2, if $\mathcal{O} \subseteq X^{3}$ is a Hurwitz orbit, where $X$ is an injective rack, then $\mathcal{O}$ has simply intersecting cycles.

Remark 4.12. For any covering $(p, S, T)$ of a $\operatorname{PSL}(2, \mathbb{Z})$-space $T$, the image of a $\sigma_{1}$-cycle in $S$ is an $x y$-cycle in $T$, and the image of a $\sigma_{2}$-cycle in $S$ is a $y x$-cycle in $T$.

Lemma 4.13. Let $T$ be a $\mathbf{P S L}(2, \mathbb{Z})$-space and let $(p, S, T)$ be a covering of $T$ with simply intersecting cycles. Let $v$ be a vertex of the graph of $T$.

(1) If there exists an $x$-loop on $v$ with label $a$, then $3 a \equiv-1(\bmod N)$.

(2) If there exists a $y$-loop on $v$ with label $a$, then $2 a \equiv 1(\bmod N)$.

Proof. It follows from Remark 4.8 .

The following four lemmas are easy consequences of the definition of simply intersecting cycles.

Lemma 4.14. Let $T$ be a $\mathbf{P S L}(2, \mathbb{Z})$-space and let $(p, S, T)$ be a covering of $T$ with simply intersecting cycles. Let $v \in T$ and $w \in C_{y x}(v) \cap C_{x y}(v)$. If $w \neq v$, then $(p, S, T)$ is not trivial.

Lemma 4.15. Let $T$ be a $\mathbf{P S L}(2, \mathbb{Z})$-space and let $(p, S, T)$ be a covering of $T$ with simply intersecting cycles. Let $v \in T$ and $N=\left|p^{-1}(v)\right|$. Let $\lambda \in \mathbb{Z}_{N}$ and $\mu \in \mathbb{Z}_{N}$ be the labels of the $x y$ - and yx-cycle containing $v$, respectively. Then $\langle\lambda\rangle \cap\langle\mu\rangle=0$ as subgroups of $\mathbb{Z}_{N}$.

Lemma 4.16. Let $T$ be a $\mathbf{P S L}(2, \mathbb{Z})$-space and let $(p, S, T)$ be a covering of $T$ with simply intersecting cycles. Let $v \in T$ and assume that $x v=v$ or $y v=v$ and that $\operatorname{PSL}(2, \mathbb{Z}) v \neq\{v\}$. Then the labels of the $x y$-and $y x$-cycles containing $v$ are 0 .

Lemma 4.17. Let $T$ be a $\mathbf{P S L}(2, \mathbb{Z})$-space and let $(p, S, T)$ be a covering of $T$ with simply intersecting cycles. Let $v, w \in T, N=\left|p^{-1}(v)\right|$ and assume that $v \neq w$ and that $v, w$ are on the same $x y$ - and the same $y x$-cycle. Let $\lambda$ and $\mu$ be the labels of the $x y$ - and $y x$-path from $v$ to $w$, respectively. Then $\lambda \not \equiv \mu(\bmod N)$.

Corollary 4.18. Let $T$ be $a \mathbf{P S L}(2, \mathbb{Z})$-space. Let $v, w \in T$ and assume that $v \neq w$, $y v=v, y w=w$ and that $v, w$ are on the same $x y$-cycle. Then $T$ has no coverings with simply intersecting cycles.

Proof. Assume to the contrary that $(p, S, T)$ is a covering of $T$ with simply intersecting cycles. Let $N=\left|p^{-1}(v)\right|$ and let $a$ and $b$ be the labels of the $y$-loops at $v$ and $w$, respectively. By Lemma $4.13(2)$ we obtain that $2 a \equiv 1(\bmod N)$ and $2 b \equiv 1(\bmod N)$ and hence $a \equiv b(\bmod N)$ and $N$ is odd. Since $y v=v$ and $y w=w$, $v$ and $w$ are on the same $y x$-cycle and the $x y$ - and $y x$-paths from $v$ to $w$ have the same labels. This is a contradiction to Lemma 4.17

\section{Cellular automata over homogeneous spaces}

As explained in the introduction, for the classification of Nichols algebras with many cubic relations we are going to determine upper bounds for immunities of Hurwitz orbits. The principle of the method to calculate these upper bounds is well-known from the theory of cellular automata. Therefore we intend to formulate our techniques in the language of cellular automata.

The following definition is inspired by [4, Definition 1.4.1], where the very similar definition of a cellular automaton over a group $G$ was given. Let $G$ be a group acting 
transitively on a set $\Omega$ and let $A$ be a set. Let $A^{\Omega}$ be the set of all functions from $\Omega$ to $A$.

Definition 5.1. Let $S$ be a set, let $\left(g_{s}\right)_{s \in S}$ be a family of elements in $G$, and let $\mu: A^{S} \rightarrow A$ be a map. Then the map $\tau: A^{\Omega} \rightarrow A^{\Omega}$ such that

$$
\tau(f)(w)=\mu\left(\left(f\left(g_{s} \cdot w\right)\right)_{s \in S}\right)
$$

for all $f \in A^{\Omega}, w \in \Omega$, is called a cellular automaton over $(G, \Omega)$ with alphabet $A$. The infinite sequence $\left(\tau^{n}(f)\right)_{n \geq 0}$ is called the evolution of $f$.

Remark 5.2. A good interpretation of a cellular automaton over $(G, \Omega)$ is the following. For any $w \in \Omega$, consider the family of points $\left(g_{s} \cdot w\right)_{s \in S}$ as the neighborhood of $w$. Then for any function $f \in A^{\Omega}$, the value of $\tau(f)$ at $w$ is obtained from the values of $f$ in the neighborhood of $w$ according to the rule determined by $\mu$.

We will only consider cellular automata over $(G, \Omega)$ with the alphabet $A=\mathbb{Z}_{2}$. For any function $f \in \mathbb{Z}_{2}^{\Omega}$ let

$$
\operatorname{supp} f=\{w \in \Omega \mid f(w)=1\} .
$$

Conversely, the characteristic function of a set $I \subseteq \Omega$ is

$$
\chi_{I} \in \mathbb{Z}_{2}^{\Omega}, \quad x \mapsto \begin{cases}1 & \text { if } x \in I, \\ 0 & \text { otherwise. }\end{cases}
$$

Definition 5.3. Let $\tau$ be a cellular automaton over $(G, \Omega)$ with alphabet $\mathbb{Z}_{2}$. We say that $\tau$ is monotonic if

(1) $\operatorname{supp} f \subseteq \operatorname{supp} \tau(f)$ for all $f \in \mathbb{Z}_{2}^{\Omega}$, and

(2) $\operatorname{supp} \tau(f) \subseteq \operatorname{supp} \tau(g)$ for all $f, g \in \mathbb{Z}_{2}^{\Omega}$ with $\operatorname{supp} f \subseteq \operatorname{supp} g$.

In what follows we will only study monotonic cellular automata over homogeneous spaces.

Definition 5.4. Let $\tau$ be a monotonic cellular automaton over $(G, \Omega)$ with alphabet $\mathbb{Z}_{2}$. For any two subsets $I, J \subseteq \Omega$ with $I \subseteq J$ we say that $I$ spreads to $J$, if $J \subseteq \operatorname{supp} \tau^{n}\left(\chi_{I}\right)$ for some $n \in \mathbb{N}$. A subset $I \subseteq \Omega$ is a quarantine if $\tau\left(\chi_{I}\right)=\chi_{I}$. A subset $I \subseteq \Omega$ is a plague if the smallest quarantine containing $I$ is $\Omega$. The cardinality of a plague $I$ is also called its size.

Remark 5.5. Let $\tau$ be a monotonic cellular automaton over $(G, \Omega)$ with alphabet $\mathbb{Z}_{2}$. If a subset $I$ spreads to another subset $J$ of $\Omega$, then any subset $I^{\prime} \subseteq \Omega$ with $I \subseteq I^{\prime}$ spreads to $J$.

Assume that $\Omega$ has only finitely many points. Then a subset $I$ of $\Omega$ is a plague if and only if it spreads to $\Omega$. In this case, any subset of $\Omega$ containing $I$ is a plague.

Plagues of Hurwitz orbits were already introduced in [6, Definition 3]. That definition is a special case of a plague of the monotonic cellular automaton in the following example, which is the main example of interest.

Example 5.6. Let $G=\mathbb{B}_{3}$ and let $\Omega$ be a finite homogeneous $G$-space. For example, if $X$ is a finite rack, then $G$ acts on $X^{3}$ by $\sigma_{1} \cdot(x, y, z)=(x \triangleright y, x, z)$ and $\sigma_{2} \cdot(x, y, z)=(x, y \triangleright z, y)$, and $\Omega$ can be taken as an orbit of this action. Let

$$
\left(g_{s}\right)_{s \in\{1, \ldots, 7\}}=\left(1, \sigma_{2}, \sigma_{1} \sigma_{2}, \sigma_{2}^{-1} \sigma_{1}^{-1}, \sigma_{1}^{-1}, \sigma_{2}^{-1}, \sigma_{1}\right) \in \mathbb{B}_{3}^{7} .
$$


As an alphabet take $A=\mathbb{Z}_{2}$. We consider the configuration given in Figure 5.1, (In Figure 5.1 the black arrow indicates the action of $\sigma_{1}$, and the dashed arrow the action of $\sigma_{2}$.) Then $x_{s}=g_{s} \cdot x_{1}$ for all $s \in\{1,2, \ldots, 7\}$. Define $\mu: A^{7} \rightarrow A$ by

$$
\begin{aligned}
\mu\left(f_{1}, f_{2}, \ldots, f_{7}\right) & =f_{1} \vee f_{2} f_{3} \vee f_{4} f_{5} \vee f_{6} f_{7} \\
& =1-\left(1-f_{1}\right)\left(1-f_{2} f_{3}\right)\left(1-f_{4} f_{5}\right)\left(1-f_{6} f_{7}\right),
\end{aligned}
$$

where $f_{1}, \ldots, f_{7} \in A$, and $\vee$ denotes logical or. Then the map $\tau$ defined by $\mu$ and $\left(g_{s}\right)_{s \in\{1, \ldots, 7\}}$ is a monotonic cellular automaton over $\left(\mathbb{B}_{3}, \Omega\right)$. A plague of this automaton is literally the same as a plague in the sense of [6. Definition 3].

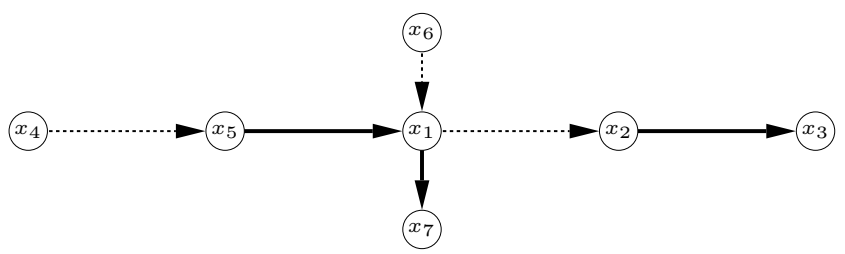

FiguRE 5.1. Neighbors of $x_{1}$

Problem 5.7. Find a plague of minimal size for the cellular automata of Example 5.6 .

Example 5.8. A cellular automaton in the very traditional sense is just a cellular automaton over $\left(\mathbb{Z}^{n}, \mathbb{Z}^{n}\right)$ with $n \in\{1,2\}$, where the action is given by the usual free action of $\mathbb{Z}^{n}$ on itself and in $\left(g_{s}\right)_{s \in S}$ only $n \in\{1,-1\} \quad\left(\left(n_{1}, n_{2}\right) \in \mathbb{Z}^{2}\right.$ with $\left|n_{1}\right|,\left|n_{2}\right| \leq 1$, respectively) appear.

5.1. Examples over $\left(\mathbb{Z}, \mathbb{Z}_{m}\right)$. In this subsection we present a family of examples of automata useful for studying the following process.

Let $m \in \mathbb{N}_{\geq 2}, f \in \mathbb{Z}_{2}^{\mathbb{Z}_{m}}, r \in \mathbb{N}$, and $a_{1}, \ldots, a_{r} \in \mathbb{Z}_{m} \backslash\{0\}$. The group $G=\mathbb{Z}$ acts transitively on $\Omega=\mathbb{Z}_{m}$. Let $A=\mathbb{Z}_{2}, S=\{0,1, \ldots, r\}$, and $\left(g_{s}\right)_{s \in S}=$ $\left(0,-a_{1},-a_{2}, \ldots,-a_{r}\right) \in G^{S}$. Define $\mu: A^{S} \rightarrow A$ by

$$
\mu\left(f_{0}, f_{1}, \ldots, f_{r}\right)= \begin{cases}1 & \text { if } f_{0}=1 \text { or } f_{1}=f_{2}=\cdots=f_{r}=1 \\ 0 & \text { otherwise }\end{cases}
$$

The map $\tau: \mathbb{Z}_{2}^{\mathbb{Z}_{m}} \rightarrow \mathbb{Z}_{2}^{\mathbb{Z}_{m}}$ defined by $\mu$ and $\left(g_{s}\right)_{s \in S}$ is then a monotonic cellular automaton. By definition,

$$
\operatorname{supp} \tau(f)=\operatorname{supp} f \cup\left\{w \in \Omega \mid f\left(x-a_{1}\right)=\cdots=f\left(x-a_{r}\right)=1\right\}
$$

for all $f \in \mathbb{Z}_{2}^{\mathbb{Z}_{m}}$. We now study plagues for special cases of this cellular automaton.

Example 5.9. Let $r=1$ and $a_{1}=\lambda$. The cellular automaton is determined by the rule

$$
\operatorname{supp} \tau(f)=\operatorname{supp} f \cup\left\{x \in \mathbb{Z}_{m} \mid f(x-\lambda)=1\right\} .
$$

Let $\Gamma=\langle\lambda\rangle$ and let $I$ be a set of representatives for $\Omega / \Gamma$. Then $I$ is a plague.

Example 5.10. Let $\lambda \in \mathbb{Z}_{m}, r=3, a_{1}=1, a_{2}=\lambda+1$, and $a_{3}=-\lambda$. Let $\Gamma=\langle\lambda\rangle$ and let $I$ be the union of a set of representatives for $\Omega / \Gamma$ with $\Gamma$. For example $I=\langle\lambda\rangle \cup\{1,2, \ldots, \lambda-1\}$. If supp $f$ contains a coset $a+\Gamma$, where $a+1 \in I$, then $\operatorname{supp} f$ spreads to $a+1+\Gamma$. Thus $I$ is a plague. 
Example 5.11. Let $\lambda \in \Omega \backslash\{0,1\}, r=2, a_{1}=\lambda, a_{2}=\lambda-1$. Let $I=\{0,1, \ldots$, $(m-1) / 2\}$ if $m$ is odd and $\{0,1, \ldots, m / 2-1\}$ if $m$ is even. Then $I$ is a plague of size $\leq(m+1) / 2$. It is in general not minimal; for example for $m \geq 3, \lambda=2$, the set $\{0,1\}$ is a plague.

\section{Plagues on Hurwitz orbits}

Example 5.6 describes cellular automata over braid group orbits. In order to prove Theorem 6.3. we have to find small plagues for these automata.

By [6, §1.4], the immunity of a Hurwitz orbit $\Sigma$ is the ratio $s /|\Sigma|$, where $s$ is the size of the smallest plague for $\Sigma$ with respect to the cellular automaton in Example 5.6. We write $\operatorname{imm}(\Sigma)$ for the immunity of $\Sigma$.

Let us formulate the cellular automaton in Example5.6 in terms of the generators $\sigma_{2}^{-1} \sigma_{1}^{-1}$ and $\sigma_{1} \sigma_{2} \sigma_{1}$ of the group $\mathbb{B}_{3}$. Note that $x=\sigma_{2}^{-1} \sigma_{1}^{-1}\langle\Delta\rangle$ and $y=\sigma_{1} \sigma_{2} \sigma_{1}\langle\Delta\rangle$ in $\operatorname{PSL}(2, \mathbb{Z})$. Let $f$ be a $\mathbb{Z}_{2}$-valued function on the braid group orbit $\Omega$ and let $P=\operatorname{supp} f$. To obtain the support of $\tau(f)$, proceed as follows.

We use the notation regarding $\Omega$ and its Schreier graph introduced in Remark4.8, Let $p$ be a point in the braid group orbit quotient $\Omega /\langle\Delta\rangle$. Let $I$ be a subset of $\mathbb{Z}_{N}$ and let $p[I]=\{p[i] \mid i \in I\}$ be the corresponding subset of the fiber over $p$. Consider the three neighboring subsets

$$
\left(\sigma_{2}^{-1} \sigma_{1}^{-1}\right)^{-1} \cdot p[I], \quad \sigma_{1} \sigma_{2} \sigma_{1} \cdot p[I], \quad \text { and } \quad \sigma_{2}^{-1} \sigma_{1}^{-1} \cdot p[I+1]
$$

of $p[I]$. They are displayed in Figure 6.1 and are denoted by $x_{1}[I-c], x_{2}[I+a]$ and $x_{3}[I+b+1]$, respectively, where, for example, $I+a$ means the translation of $I$ by $a$, i.e. $I+a=\{i+a \mid i \in I\}$. In this setting we call $p[I]$ a pivot.

By Example[5.6, if $P$ contains the subsets $x_{1}[I-c]=\sigma_{1} \sigma_{2} \cdot p[I]$ and $\sigma_{2} \cdot x_{1}[I-c]=$ $x_{2}[I+a]$, then $\operatorname{supp} \tau(f)$ contains

$$
\sigma_{1} \cdot x_{2}[I+a]=\sigma_{1} \sigma_{2} \sigma_{1} \sigma_{2} \cdot p[I]=\sigma_{2}^{-1} \sigma_{1}^{-1} \Delta \cdot p[I]=x_{3}[I+b+1] .
$$

Similarly, if any two of the neighboring subsets $x_{1}[I-c], x_{2}[I+a]$, and $x_{3}[I+b+1]$ of $p[I]$ are contained in $P$, then the third is a subset of $\operatorname{supp} \tau(f)$. Moreover, $\operatorname{supp} \tau(f)$ is the smallest subset of $\Omega$ containing supp $f$ and all sets constructed this way for some point $p$ and some subset $I \subseteq \mathbb{Z}_{N}$.

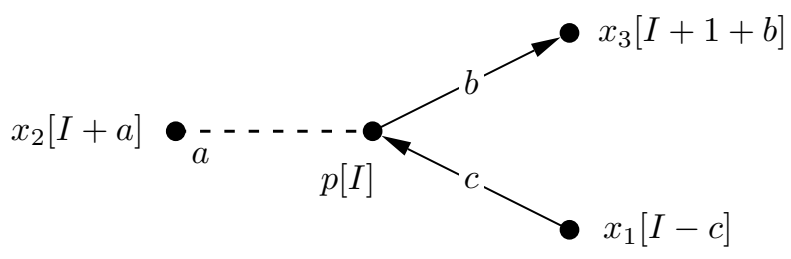

FiguRE 6.1. The cellular automaton on braid group orbits

We intend to obtain an upper bound for the immunity of each Hurwitz orbit using the cycle structure at each vertex. For that purpose, we define

$$
\left(\omega_{i j}^{\prime}\right)_{i, j \geq 1}=\left(\begin{array}{cccccc}
1 & 1 / 3 & 11 / 24 & 1 / 2 & 1 / 2 & \ldots \\
1 / 3 & 1 / 3 & 1 / 3 & 1 / 3 & 1 / 3 & \ldots \\
11 / 24 & 1 / 3 & 7 / 24 & 7 / 24 & 7 / 24 & \ldots \\
1 / 2 & 1 / 3 & 7 / 24 & 1 / 4 & 1 / 4 & \ldots \\
1 / 2 & 1 / 3 & 7 / 24 & 1 / 4 & 1 / 4 & \ldots \\
\vdots & \vdots & \vdots & \vdots & \vdots & \ddots
\end{array}\right) .
$$


The notation for $\mathbf{P S L}(2, \mathbb{Z})$-spaces was fixed below Proposition 4.3. For the coverings of a $\operatorname{PSL}(2, \mathbb{Z})$-space $\Sigma_{*}$, where $*$ stands for any possible index, we use the notation $\Sigma_{*}^{N ; a, b, \ldots}$. Here, $N$ is the size of the fiber over a point of $\Sigma_{*}$, and $a, b, \ldots$ are the values of the individual labels determining the covering. These labels are chosen by the method described in Remark 4.8 and can be read off the corresponding figure. We again use the same notation for the homogeneous space and its Schreier graph.

Let $\Sigma$ be a homogeneous $\mathbb{B}_{3}$-space and let $v \in \Sigma$. Assume that $v$ belongs to a $\sigma_{1}$-cycle of length $i$, and also to a $\sigma_{2}$-cycle of length $j$. Then we write $c(v)=(i, j)$. Let $\omega: \Sigma \rightarrow \mathbb{Q}$ be the map defined by

$$
\omega(v)= \begin{cases}\omega_{i j}^{\prime}+\frac{1}{30}=\frac{13}{40} & \text { if } \Sigma=\Sigma_{4 A}^{5 ; 3,2} \text { and } v \in v_{1}[*], \\ \omega_{i j}^{\prime}+\frac{1}{12}=\frac{1}{3} & \text { if } \Sigma=\Sigma_{6 A}^{4 ; 2,2} \text { and } v \in v_{3}[*], \\ \omega_{i j}^{\prime}+\frac{1}{24}=\frac{1}{3} & \text { if } \Sigma \text { is the trivial covering of } \Sigma_{12 C}, \\ \omega_{i j}^{\prime} & \text { otherwise, }\end{cases}
$$

where $c(v)=(i, j)$, and $v_{i}[*]$ is defined in Remark 4.8.

Remark 6.1. If we would not add the three exceptional coverings in the definition of $\omega$, then Theorem 6.3 below would not hold. We could compensate by changing the infinite matrix $\left(\omega_{i j}^{\prime}\right)_{i, j \geq 1}$, but then we would have only a weak upper bound for the immunity of a Hurwitz orbit. In that case we would not have enough information to reduce the proof of Theorem 9.3 to the study of a few small racks.

Definition 6.2. Let $\Sigma$ be a homogeneous $\mathbb{B}_{3}$-space. The weight of $\Sigma$ is defined as

$$
\omega(\Sigma)=\frac{1}{|\Sigma|} \sum_{v \in \Sigma} \omega(v) .
$$

The main goal of this section is to formulate a good upper bound for the immunity of Hurwitz orbits.

Theorem 6.3. Let $\Sigma$ be a covering with simply intersecting cycles of a finite homogeneous $\operatorname{PSL}(2, \mathbb{Z})$-space $\bar{\Sigma}$. Assume that any $x y$-cycle of $\bar{\Sigma}$ has at most four elements. Then $\operatorname{imm}(\Sigma) \leq \omega(\Sigma)$.

We prove Theorem 6.3 in Section 7

\section{Proof of Theorem 6.3}

This section contains a case-by-case analysis of the coverings, immunities and weights for the Schreier graphs of finite homogeneous $\operatorname{PSL}(2, \mathbb{Z})$-spaces $\bar{\Sigma}$ such that any $x y$-cycle of $\bar{\Sigma}$ has at most four elements. The main goal of this section is to prove Theorem 6.3

\subsection{The graph $\Sigma_{1 A}$.}

Lemma 7.1. Every covering of $\Sigma_{1 A}$ in Figure 7.1 with simply intersecting cycles is trivial.

Proof. Lemma4.13implies that $3 a \equiv-1(\bmod N)$ and $2 b \equiv 1(\bmod N)$. By Lemma 4.16. $a+b \equiv 0(\bmod N)$ and hence $N=1$. 


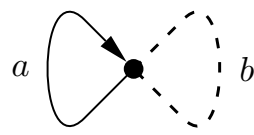

FiguRE 7.1. Schreier graph $\Sigma_{1 A}$ and its coverings

The following lemma is trivial, but we state it for completeness.

Lemma 7.2. Let $\Sigma$ be the trivial covering of the homogeneous $\mathbf{P S L}(2, \mathbb{Z})$-space $\Sigma_{1 A}$. Then $\operatorname{imm}(\Sigma)=1=\omega(\Sigma)$.

\subsection{The graph $\Sigma_{2 A}$.}

Lemma 7.3. The graph $\Sigma_{2 A}$ in Figure 7.2 has no coverings with simply intersecting cycles.

Proof. Lemma 4.13 implies that $3 a \equiv 3 b \equiv-1(\bmod N)$. Further, from Lemma 4.16 on $v$ we obtain $a+b+1 \equiv 0(\bmod N)$ and the claim follows.

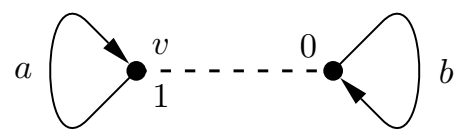

Figure 7.2. Schreier graph $\Sigma_{2 A}$ and its coverings

\subsection{The graph $\Sigma_{3 A}$.}

Lemma 7.4. Any covering of $\Sigma_{3 A}$ in Figure 7.3 with simply intersecting cycles satisfies $2 a \equiv 1(\bmod N), a \equiv b(\bmod N)$.

Proof. Lemma 4.13 on $v_{1}$ implies that $2 a \equiv 1(\bmod N)$. Since $v_{1}$ belongs to cycles with label $a-b, a \equiv b(\bmod N)$ by Lemma 4.16 .

Example 7.5. The trivial covering of $\Sigma_{3 A}$ in Figure 7.3 is isomorphic to the Hurwitz orbit with three elements of [6, Figure 8].

Lemma 7.6. Let $\Sigma$ be a covering of $\Sigma_{3 A}$ with simply intersecting cycles. Then $\operatorname{imm}(\Sigma) \leq 1 / 3=\omega(\Sigma)$.

Proof. First we prove that $P=v_{2}[*]$ is a plague. With $v_{3}[*]$ as a pivot we see that $P$ spreads to $v_{1}[*]$. Then, with $v_{1}[*]$ as a pivot, it also spreads to $v_{3}[*]$ and hence $\operatorname{imm}(\Sigma) \leq 1 / 3$. Now we prove $\omega(\Sigma)=1 / 3$. By Lemma 7.4 the cycle structure on each vertex of $\Sigma$ is the following:

$$
v_{1}[i] \text { : two 2-cycles, }
$$

$v_{2}[i]$ and $v_{3}[i]$ : one 2-cycle and a cycle of length $|\langle b\rangle|$,

for all $i \in \mathbb{Z}_{N}$. Then $\omega(\Sigma)=1 / 3$ and this proves the claim. 


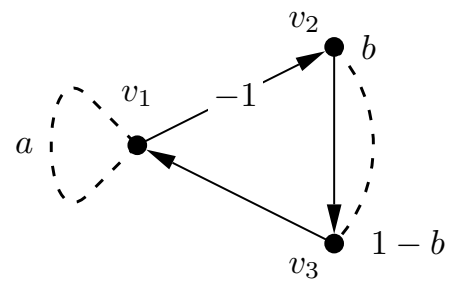

FiguRE 7.3. Schreier graph $\Sigma_{3 A}$ and its coverings

\subsection{The graph $\Sigma_{3 B}$.}

Lemma 7.7. The graph $\Sigma_{3 B}$ in Figure 7.4 has no coverings with simply intersecting cycles.

Proof. This follows from Corollary 4.18 ,

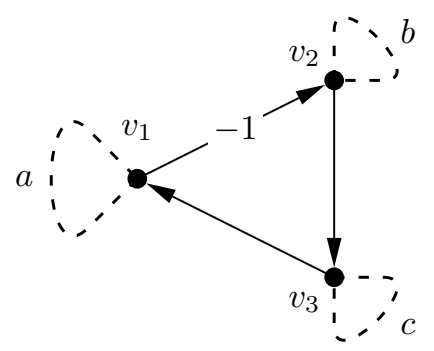

FiguRE 7.4. Schreier graph $\Sigma_{3 B}$ and its coverings

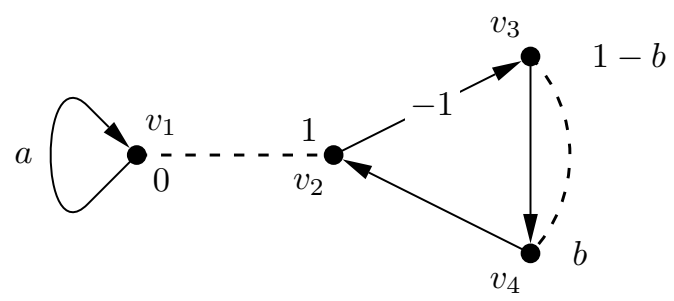

Figure 7.5. Schreier graph $\Sigma_{4 A}$ and its coverings

\subsection{The graph $\Sigma_{4 A}$.}

Lemma 7.8. Any covering of $\Sigma_{4 A}$ in Figure 7.5 with simply intersecting cycles satisfies $3 a \equiv-1(\bmod N), a+b \equiv 0(\bmod N)$ and $N>1$. 
Proof. Since $v_{2} \in C_{x y}\left(v_{1}\right) \cap C_{y x}\left(v_{1}\right)$, Lemma 4.14 implies that $N>1$. Since $v_{1}$ is on an $x y$-cycle with label $a+b$, by Lemmas 4.13(1) and 4.16 on $v_{1}$ the claim follows.

Example 7.9. Let $\Sigma$ be the covering of $\Sigma_{4 A}$ with $N=2$ and $a=b=1$. Then $\Sigma$ can be presented as

$$
\sigma_{1}=\left(v_{1} v_{3} \Delta v_{2}\right)\left(\Delta v_{1} \Delta v_{3} v_{2}\right), \quad \sigma_{2}=\left(v_{1} v_{2} v_{4}\right)\left(\Delta v_{1} \Delta v_{2} \Delta v_{4}\right),
$$

and $\Sigma$ is isomorphic to the Hurwitz orbit of eight elements of [6, Figure 10]. The isomorphism is given by the bijection

$$
\left(\begin{array}{cccccccc}
v_{1} & \Delta v_{1} & v_{2} & \Delta v_{2} & v_{3} & \Delta v_{3} & v_{4} & \Delta v_{4} \\
E & D & B & G & H & A & C & F
\end{array}\right)
$$

Lemma 7.10. Let $\Sigma$ be a covering of $\Sigma_{4 A}$ with simply intersecting cycles. Then $\operatorname{imm}(\Sigma) \leq \frac{N+1}{4 N}$

Proof. Let $I=\{0\} \subseteq \mathbb{Z}_{N}$. We claim that $P=v_{3}[*] \cup v_{1}[I]$ is a plague. With $v_{4}[*]$ as a pivot, $P$ spreads to $v_{2}[*]$. Since the neighbors of $v_{1}[\lambda]$ are $v_{1}[\lambda-a], v_{1}[\lambda+a+1]$ and $v_{2}[\lambda+1]$, pivoting from $v_{1}[I+a]$ implies that $P$ spreads to $v_{1}[I+2 a+1]$. We describe this situation with the following table:

$$
\begin{array}{c|cc}
\text { pivot } & v_{4}[*] & v_{1}[I+a] \\
\hline & v_{2}[*] & v_{1}[I+2 a+1]
\end{array}
$$

From Lemma 7.8 we obtain that $\langle 2 a+1\rangle=\langle-a\rangle=\mathbb{Z}_{N}$, and hence Example 5.9 implies that $P$ spreads to $v_{1}[*]$. Now with $v_{2}[*]$ as a pivot we see that $P$ spreads to $\Sigma$ and we are done. Thus the claim follows.

Lemma 7.11. Let $\Sigma$ be a covering of $\Sigma_{4 A}$ with simply intersecting cycles. Then $\operatorname{imm}(\Sigma) \leq \omega(\Sigma)$.

Proof. By Lemma 7.8 the cycle structure on each vertex of $\Sigma$ is the following:

$$
\begin{aligned}
& v_{1}[i] \text { and } v_{2}[i]: \text { two } 3 \text {-cycles, } \\
& v_{3}[i] \text { and } v_{4}[i]: \text { one } 3 \text {-cycle and a cycle of length }|\langle 1-b\rangle|,
\end{aligned}
$$

for all $i \in \mathbb{Z}_{N}$. Assume first that $N=3 k+1$. Then $a=k, b=2 k+1$ and $k \geq 1$. Since $3(b-1) \equiv-2(\bmod N)$ and $b-1$ is even, we get

$$
|\langle 1-b\rangle|=|\langle 2\rangle|= \begin{cases}N & \text { if } k \text { is even, } \\ \frac{N}{2} & \text { if } k \text { is odd. }\end{cases}
$$

By (7.2),$\omega\left(v_{3}[i]\right)=\omega\left(v_{4}[i]\right)=1 / 3$ if $k=1$, and $\omega\left(v_{3}[i]\right)=\omega\left(v_{4}[i]\right)=7 / 24$ if $k>1$, for all $i \in \mathbb{Z}_{N}$. Therefore

$$
\omega(\Sigma)= \begin{cases}5 / 16 & \text { if } k=1 \\ 7 / 24 & \text { if } k>1\end{cases}
$$

If $k=1$, then $N=4$ and $\operatorname{imm}(\Sigma) \leq \frac{N+1}{4 N}=5 / 16=\omega(\Sigma)$. Otherwise, if $k>1$, then $N>6$ and hence

$$
\operatorname{imm}(\Sigma) \leq \frac{N+1}{4 N}<\frac{7}{24}=\omega(\Sigma) .
$$


Now assume that $N=3 k+2$. Then $a=2 k+1, b=k+1$ and $k \geq 0$. Further, $3(b-1) \equiv-2(\bmod N)$ and hence

$$
|\langle 1-b\rangle|=\left\{\begin{array}{cl}
\frac{N}{2} & \text { if } k \text { is even } \\
N & \text { if } k \text { is odd. }
\end{array}\right.
$$

By (7.2),

$$
\omega\left(v_{3}[i]\right)= \begin{cases}11 / 24 & \text { if } k=0, \\ 7 / 24 & \text { if } k>0,\end{cases}
$$

for all $i \in \mathbb{Z}_{N}$, and therefore

$$
\omega(\Sigma)= \begin{cases}3 / 8 & \text { if } k=0, \\ 7 / 24 & \text { if } k>0 .\end{cases}
$$

If $k=0$, then $N=2$ and $\operatorname{imm}(\Sigma) \leq \frac{N+1}{4 N}=3 / 8=\omega(\Sigma)$. If $k=1$, then $N=5$,

$$
\operatorname{imm}(\Sigma) \leq \frac{N+1}{4 N}=3 / 10=(4 \cdot 7 / 24+1 / 30) / 4=\omega(\Sigma) .
$$

Finally, if $k>1$, then $\operatorname{imm}(\Sigma) \leq \frac{N+1}{4 N} \leq 7 / 24=\omega(\Sigma)$ for $N \geq 6$.

Example 7.12. Let $x_{1}=(12345), x_{2}=(12453)$ and $x_{3}=(14352)$ be 5 -cycles in $\mathbb{A}_{5}$. Let $X$ be the rack associated to the conjugacy class of $x_{1}$ in $\mathbb{A}_{5}$. The covering $\Sigma_{4 A}^{5 ; 3,2}$ can be realized as the Hurwitz orbit of $\left(x_{1}, x_{2}, x_{3}\right) \in X^{3}$.

Lemma 7.13. Let $X$ be an injective indecomposable rack. Assume that $X^{3}$ contains a Hurwitz orbit isomorphic to the covering $\Sigma_{4 A}^{5 ; 3,2}$. If $(x, y, z) \in v_{1}[*]$, then $(x \triangleright y) \triangleright y$ $=z$.

Proof. By inspection,

$$
\begin{aligned}
\sigma_{1} v_{1}[i] & =v_{3}[i], & \sigma_{2} v_{1}[i] & =v_{2}[i+4], \\
\sigma_{1} v_{2}[i] & =v_{1}[i+3], & \sigma_{2} v_{2}[i] & =v_{4}[i+1], \\
\sigma_{1} v_{3}[i] & =v_{2}[i+2], & \sigma_{2} v_{3}[i] & =v_{3}[i+4], \\
\sigma_{1} v_{4}[i] & =v_{4}[i+4], & \sigma_{2} v_{4}[i] & =v_{1}[i] .
\end{aligned}
$$

Let $1,2,3 \in X$ with $v_{1}[0]=(1,2,3) \in \Sigma_{4 A}^{5 ; 3,2} \subseteq X^{3}$. Set $1 \triangleright 2=4,1 \triangleright 3=5$ and $1 \triangleright 4=6$. (The elements $1,2,3,4,5,6 \in X$ are not necessarily pairwise distinct.) We claim that $(1 \triangleright 2) \triangleright 1=2$. Indeed, using (7.3) we obtain $v_{3}[0]=\sigma_{1} v_{1}[0]=(1 \triangleright 2,1,3)$. By (7.5),$v_{2}[2]=\sigma_{1} v_{3}[0]=((1 \triangleright 2) \triangleright 1,1 \triangleright 2,3)$. Since $v_{1}[0]=\sigma_{1} v_{2}[2]$, we obtain $(1 \triangleright 2) \triangleright 1=2$. Similarly, using formulas (7.3)-(7.6), straightforward computations show that:

\begin{tabular}{c|c|c|c|c}
$i$ & $v_{1}[i]$ & $v_{2}[i]$ & $v_{3}[i]$ & $v_{4}[i]$ \\
\hline 0 & $(1,2,3)$ & $(3,4,5)$ & $(4,1,3)$ & $(1,3,4)$ \\
1 & $(3,5,6)$ & $(6,4,1)$ & $(4,3,6)$ & $(3,6,4)$ \\
2 & $(6,1,2)$ & $(2,4,3)$ & $(4,6,2)$ & $(6,2,4)$ \\
3 & $(2,3,5)$ & $(5,4,6)$ & $(4,2,5)$ & $(2,5,4)$ \\
4 & $(5,6,1)$ & $(1,4,2)$ & $(4,5,1)$ & $(5,1,4)$
\end{tabular}

Since $\sigma_{2} \cdot(1,4,2)=\sigma_{2} v_{2}[4]=v_{4}[0]=(1,3,4)$, we get $(1 \triangleright 2) \triangleright 2=4 \triangleright 2=3$. Since $\Delta$ acts transitively on $v_{1}[*]$, the lemma follows. 
7.6. The graph $\Sigma_{4 B}$.

Lemma 7.14. The graph $\Sigma_{4 B}$ in Figure 7.6 has no coverings with simply intersecting cycles.

Proof. This follows from Corollary 4.18 .

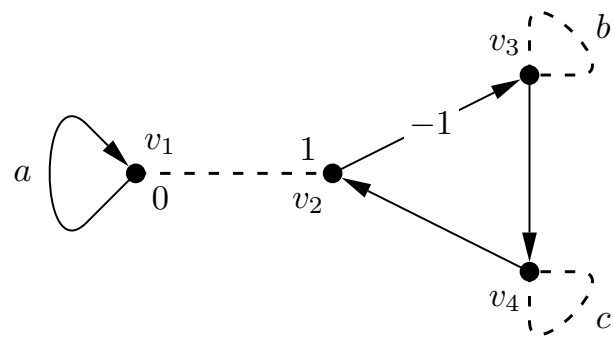

FiguRE 7.6. Schreier graph $\Sigma_{4 B}$ and its coverings

\subsection{The graph $\Sigma_{6 A}$.}

Lemma 7.15. Any covering of $\Sigma_{6 A}$ in Figure 7.7 with simply intersecting cycles satisfies $N \neq 1, a+b \equiv 0(\bmod N)$ and $2 b \not \equiv 1(\bmod N)$.

Proof. The $x y$-cycles and their labels are: $\left(v_{1} v_{3} v_{5} v_{4}\right)$ with $a+b,\left(v_{2}\right)$ with $-a$ and $\left(v_{6}\right)$ with $-b+1$. The $y x$-cycles are: $\left(v_{2} v_{4} v_{6} v_{3}\right)$ with $a+b,\left(v_{5}\right)$ with $-b+1$ and $\left(v_{1}\right)$ with $-a$. Lemma 4.15 on $v_{3}$ implies $a+b \equiv 0(\bmod N)$. Since $v_{3}$ and $v_{4}$ are on the same cycle, $N>1$ by Lemma 4.14 and $a+1 \not \equiv b(\bmod N)$ by Lemma 4.17.

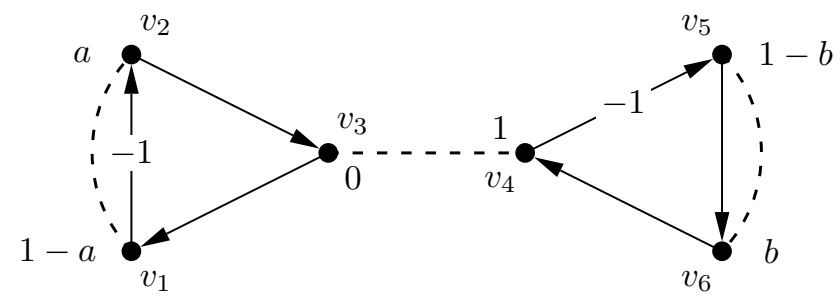

FIGURE 7.7. Schreier graph $\Sigma_{6 A}$ and its coverings

Lemma 7.16. Let $\Sigma$ be a covering of $\Sigma_{6 A}$ with simply intersecting cycles, and let

$$
m=\min \left\{\frac{|\langle 1+a\rangle|-1}{6 N}, \frac{1}{6|\langle-a\rangle|}\right\} \text {. }
$$

Then

$$
\operatorname{imm}(\Sigma) \leq \begin{cases}1 / 3 & \text { if } a \in\{0,-1\} \\ \frac{1}{6|\langle a+1\rangle|}+\frac{1}{6}+m & \text { otherwise. }\end{cases}
$$


Proof. Let $\Sigma=\Sigma_{6 A}^{N ; a, b}$ as in Figure 7.7. There exists a unique isomorphism from $\Sigma$ to $\Sigma_{6 A}^{N ; b-1,1+a}$ with

$$
\begin{array}{llll}
v_{1} \mapsto v_{5}, & v_{3} \mapsto v_{4}[1], & & v_{5} \mapsto v_{1}[1], \\
v_{2} \mapsto v_{6}[1], & v_{4} \mapsto v_{3}, & v_{6} \mapsto v_{2} .
\end{array}
$$

The labels $-a$ and $1-b$ are permuted under this isomorphism. Hence without loss of generality we may assume $|\langle-a\rangle| \leq|\langle 1-b\rangle|$. By Lemma 7.15 we have $b \equiv-a(\bmod N)$, and the cycle structure of $\Sigma$ is the following:

$$
\begin{aligned}
& v_{1}[i] \text { and } v_{2}[i]: \text { one } 4 \text {-cycle and a cycle of length }|\langle-a\rangle|, \\
& v_{3}[i] \text { and } v_{4}[i]: \text { two } 4 \text {-cycles, } \\
& v_{5}[i] \text { and } v_{6}[i]: \text { one } 4 \text {-cycle and a cycle of length }|\langle 1+a\rangle|,
\end{aligned}
$$

for all $i \in \mathbb{Z}_{N}$. First assume that $a \in\{0,-1\}$. A straightforward computation shows that $v_{1}[*] \cup v_{5}[*]$ is a plague of size $2 N$. In this case, $\operatorname{imm}(\Sigma) \leq 1 / 3$.

Now assume that $a \notin\{0,-1\}$. Let $I$ (resp. $J$ ) be a set of representatives for $\mathbb{Z}_{N} /\langle-a\rangle$ (resp. $\mathbb{Z}_{N} /\langle a+1\rangle$ ). We claim that $P=v_{1}[*] \cup v_{2}[I] \cup v_{5}[J]$ is a plague.

First we compute

$$
\begin{array}{c|cc}
\text { pivot } & v_{2}[*] & v_{1}[I] \\
\hline & v_{3}[*] & v_{2}[I+a]
\end{array}
$$

and hence $P$ spreads to $v_{2}[*]$ by Example [5.9. Now we compute

$$
\begin{array}{c|cc}
\text { pivot } & v_{3}[*] & v_{6}[J] \\
\hline & v_{4}[*] & v_{5}[J+1+a]
\end{array}
$$

and hence $P$ spreads to $v_{1}[*] \cup v_{2}[*] \cup v_{3}[*] \cup v_{4}[*] \cup v_{5}[*]$ by Example [5.9. Then we conclude that $P$ is a plague.

As an alternative, let $J$ be a set of representatives for $\mathbb{Z}_{N} /\langle 1+a\rangle$, and let $K=\langle 1+a\rangle$ and $I=J \cup K$. We prove that $P=v_{1}[*] \cup v_{5}[I]$ is a plague. With $v_{2}[*]$ as a pivot we see that $P$ spreads to $v_{3}[*]$, with $v_{4}[I]$ it spreads to $v_{6}[I]$, and with $v_{5}[(I-1) \cap(I+a)]$ it spreads further to $v_{4}[I \cap(I+a+1)]$. Finally, with $v_{6}[(I-1) \cap(I+a) \cap(I-a-1)]$ as a pivot, $P$ further spreads to $v_{5}[(I-1) \cap$ $(I+a) \cap(I-a-1)]$. By restricting our attention to the fiber over $v_{5}$, we conclude from Example 5.10 that $P$ spreads to $v_{5}[*]$. Since $v_{1}[*] \cup v_{5}[*]$ is a plague of $\Sigma$, the claim of the lemma follows.

Lemma 7.17. Let $\Sigma$ be a covering of $\Sigma_{6 A}$ with simply intersecting cycles. Then $\operatorname{imm}(\Sigma) \leq \omega(\Sigma)$.

Proof. By Lemma 7.15 we have $b \equiv-a(\bmod N)$ and $N>1$. As in the proof of Lemma 7.16, we may assume $|\langle-a\rangle| \leq|\langle 1+a\rangle|$. We split the proof into several cases according to the order of $\langle-a\rangle$. Assume first that $|\langle-a\rangle|=1$. Then $a=0$ and $|\langle 1+a\rangle|=N \geq 2$. Hence

$$
\omega(\Sigma) \geq 2(1 / 2+1 / 4+1 / 4) / 6=1 / 3=\operatorname{imm}(\Sigma)
$$

by Lemma 7.16 . 
Now assume that $|\langle-a\rangle|=2$. Then $a=N / 2, N$ is even, and $N \geq 4$. (If $N=2$, then $a=1$, which contradicts $|\langle-a\rangle| \leq|\langle 1+a\rangle|$.) As before, we need to consider three cases: $N=4, N=6$ and $N \geq 8$. Then

\begin{tabular}{c|c|c|c|c}
$N$ & $|\langle 1+a\rangle|$ & $\omega\left(v_{1}\right), \omega\left(v_{2}\right)$ & $\omega\left(v_{3}\right), \omega\left(v_{4}\right)$ & $\omega\left(v_{5}\right), \omega\left(v_{6}\right)$ \\
\hline 4 & 4 & $1 / 3$ & $1 / 4$ & $1 / 4$ \\
6 & 3 & $1 / 3$ & $1 / 4$ & $7 / 24$ \\
$\geq 8$ & $\geq 5$ & $1 / 3$ & $1 / 4$ & $1 / 4$
\end{tabular}

and therefore (observe that the case $N=4$ is exceptional)

$$
\omega(\Sigma)= \begin{cases}(3 \cdot 1 / 3+3 \cdot 1 / 4) / 6=7 / 24 & \text { if } N=4 \\ 7 / 24 & \text { if } N=6 \\ 5 / 18 & \text { if } N \geq 8\end{cases}
$$

Further, $|\langle a+1\rangle|=N / 2$ if $a$ is odd and $|\langle a+1\rangle|=N$ if $a$ is even. Thus Lemma 7.16 yields that $\operatorname{imm}(\Sigma) \leq 7 / 24$ for $N=4, \operatorname{imm}(\Sigma) \leq 1 / 4+1 / 6 N$ for $N \geq 6$, both if $a$ is odd and if $a$ is even. This implies the claim.

Now assume that $|\langle-a\rangle|=3$. Clearly, $3 a \equiv 0(\bmod N)$ and 3 divides $N$. We claim that $4 \leq|\langle 1+a\rangle|$. Indeed, if $|\langle 1+a\rangle|=3$, then $3(1+a) \equiv 0(\bmod N)$ and $N=3, a=2, b=1$, by Lemma 7.15 . Hence $|\langle 1+a\rangle|=0$, which is a contradiction. Therefore, by (7.7)- (7.9) and Lemma 7.16, we obtain $\operatorname{imm}(\Sigma) \leq 19 / 72=\omega(\Sigma)$.

Finally, assume that $|\langle-a\rangle| \geq 4$. Using (7.7)-(7.9) and Lemma 7.16 we obtain $\omega(\Sigma)=1 / 4 \geq \operatorname{imm}(\Sigma)$.

Example 7.18. Let $X=\operatorname{Aff}(5,2)$ or $X=\operatorname{Aff}(5,3)$. The Hurwitz orbit of $(1,1,2) \in X^{3}$ is isomorphic to the covering $\Sigma_{6 A}^{4 ; 2,2}$.

Lemma 7.19. Let $X$ be an injective indecomposable rack and $x \in X$. Assume that $X$ has at least one Hurwitz orbit isomorphic to the covering $\Sigma_{6 A}^{4 ; 2,2}$. Then

(1) $\varphi_{x}$ contains a 4 -cycle and $k_{4} \geq 4$.

(2) If $(x, y, z) \in v_{3}[*]$ and $w=x \triangleright y$, then $w \triangleright((w \triangleright x) \triangleright x)=z$.

Proof. We may assume that $X=\{1,2, \ldots, d\}$. By inspection,

$$
\begin{aligned}
\sigma_{1} v_{1}[i] & =v_{3}[i+2], & \sigma_{2} v_{1}[i] & =v_{1}[i+2], \\
\sigma_{1} v_{2}[i] & =v_{2}[i+2], & \sigma_{2} v_{2}[i] & =v_{4}[i+1], \\
\sigma_{1} v_{3}[i] & =v_{5}[i], & \sigma_{2} v_{3}[i] & =v_{2}[i+2], \\
\sigma_{1} v_{4}[i] & =v_{1}[i], & \sigma_{2} v_{4}[i] & =v_{6}[i+1], \\
\sigma_{1} v_{5}[i] & =v_{4}[i+2], & \sigma_{2} v_{5}[i] & =v_{5}[i+3], \\
\sigma_{1} v_{6}[i] & =v_{6}[i+3], & \sigma_{2} v_{6}[i] & =v_{3}[i] .
\end{aligned}
$$

Without loss of generality we may assume that $v_{1}[0]=(1,2,3) \in X^{3}$, where $|\{1,2,3\}| \leq 3$. Set $4=1 \triangleright 2,5=1 \triangleright 4,6=4 \triangleright 5,7=2 \triangleright 4,8=7 \triangleright 1$ and $9=\varphi_{2}^{-1}(8)$. Using formulas (7.10) (7.15), a straightforward computation shows that:

\begin{tabular}{c|c|c|c|c|c|c}
$i$ & $v_{1}[i]$ & $v_{2}[i]$ & $v_{3}[i]$ & $v_{4}[i]$ & $v_{5}[i]$ & $v_{6}[i]$ \\
\hline 0 & $(1,2,3)$ & $(4,7,1)$ & $(7,1,2)$ & $(2,8,3)$ & $(8,7,2)$ & $(7,2,8)$ \\
1 & $(9,4,7)$ & $(3,2,9)$ & $(2,9,4)$ & $(4,8,7)$ & $(8,2,4)$ & $(2,4,8)$ \\
2 & $(1,3,2)$ & $(7,4,1)$ & $(4,1,3)$ & $(3,8,2)$ & $(8,4,3)$ & $(4,3,8)$ \\
3 & $(9,7,4)$ & $(2,3,9)$ & $(3,9,7)$ & $(7,8,4)$ & $(8,3,7)$ & $(3,7,8)$
\end{tabular}


Since there are no loops, we obtain $|\{2,3,4,7\}|=4$. Furthermore, using (7.10)(17.15) we obtain that $\varphi_{8}=\left(\begin{array}{ll}2 & 734\end{array}\right) \tau$ for some $\tau \in \mathbb{S}_{d},\left.\tau\right|_{\{2,3,4,7\}}=$ id. In particular,

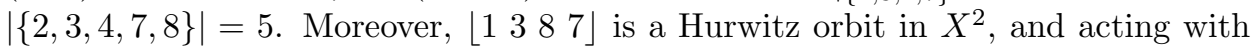

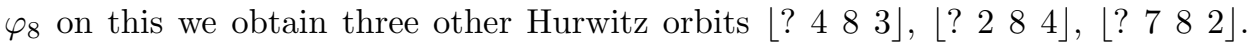
Hence $k_{4} \geq 4$. Finally, $3 \triangleright 7=4,7 \triangleright 1=8,8 \triangleright((8 \triangleright 7) \triangleright 7)=8 \triangleright(3 \triangleright 7)=8 \triangleright 4=2$, and hence $w \triangleright((w \triangleright x) \triangleright x)=z$ for $(x, y, z)=(7,1,2)$.

\subsection{The graph $\Sigma_{6 B}$.}

Lemma 7.20. There is no covering of $\Sigma_{6 B}$ in Figure 7.8 with simply intersecting cycles.

Proof. The $x y$-cycles and their labels are $\left(v_{1} v_{2} v_{5}\right)$ with $a-c$ and $\left(v_{3} v_{6} v_{4}\right)$ with $c+b$. The $y x$-cycles and their labels are $\left(v_{1} v_{4} v_{3}\right)$ with $a-c$ and $\left(v_{2} v_{5} v_{6}\right)$ with $c+b$. By Lemma 4.14 on $v_{3}$ and $v_{4}, N>1$. Lemma 4.13 on $v_{1}$ and $v_{6}$ implies that $2 a \equiv 2 b \equiv 1(\bmod N)$ and hence $a \equiv b(\bmod N)$ and $N$ is odd. By Lemma 4.16 on $v_{1}$ and $v_{6}, b+c \equiv 0(\bmod N)$ and $a-c \equiv 0(\bmod N)$. Then $a \equiv-b(\bmod N)$ and hence $1 \equiv-1(\bmod N)$. This is a contradiction to $N>2$.

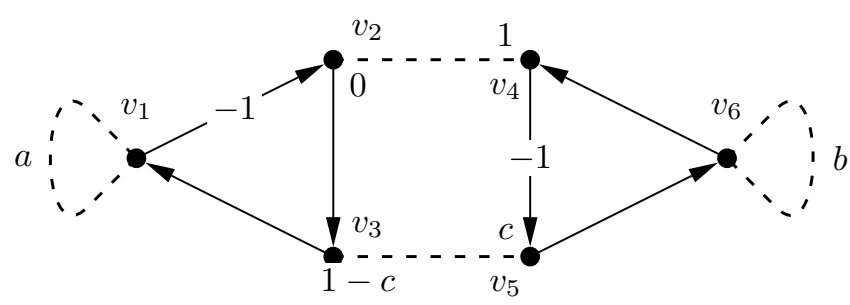

FiguRE 7.8. Schreier graph $\Sigma_{6 B}$ and its coverings

\subsection{The graph $\Sigma_{6 C}$.}

Lemma 7.21. There is no covering of $\Sigma_{6 C}$ in Figure 7.9 with simply intersecting cycles.

Proof. This follows from Corollary 4.18 ,

\subsection{The graph $\Sigma_{6 D}$.}

Lemma 7.22. Let $\Sigma$ be a covering of $\Sigma_{6 D}$ in Figure 7.10 with simply intersecting cycles. Then
(1) $\langle a+b\rangle \cap\langle-a+1\rangle=0$,
(2) $\langle a+b\rangle \cap\langle-b\rangle=0$,
(3) $\langle-a+1\rangle \cap\langle-b\rangle=0$.

Proof. The $x y$-cycles and their labels are: $\left(v_{1} v_{4}\right)$ with label $a+b,\left(\begin{array}{ll}v_{2} & v_{6}\end{array}\right)$ with $-a+1$ and $\left(v_{3} v_{5}\right)$ with $-b$. The $y x$-cycles are: $\left(v_{1} v_{5}\right)$ with label $-a+1,\left(v_{2} v_{4}\right)$ with $-b$ and $\left(v_{3} v_{6}\right)$ with $a+b$. Now apply Lemma 4.15. 


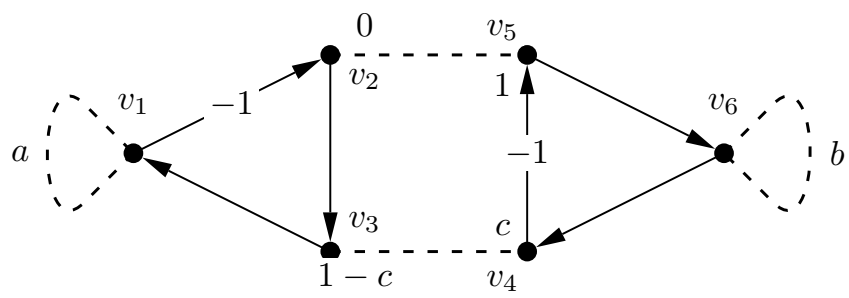

FiguRE 7.9. Schreier graph $\Sigma_{6 C}$ and its coverings

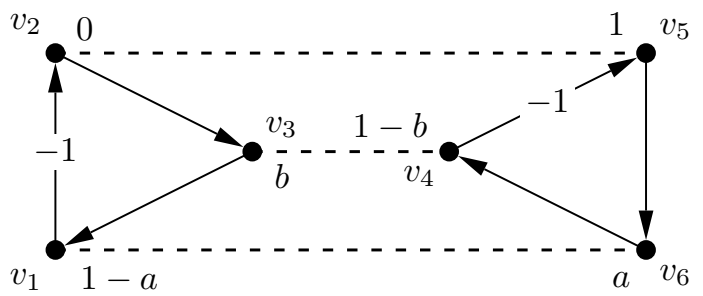

Figure 7.10. Schreier graph $\Sigma_{6 D}$ and its coverings

Example 7.23. The trivial covering of $\Sigma_{6 D}$ is isomorphic to the Hurwitz orbit of six elements of [6, Figure 9].

Lemma 7.24. Let $\Sigma$ be a covering of $\Sigma_{6 D}$ with simply intersecting cycles. Let $c \in\{1-a,-b, a+b\}$ and let $n=|\langle c\rangle|$. Then

$$
\operatorname{imm}(\Sigma) \leq \frac{N+N / n+n-1}{6 N} .
$$

Proof. There exists an isomorphism $\Sigma_{6 D}^{N ; a, b} \rightarrow \Sigma_{6 D}^{N ; 1-a-b, a-1}, v_{1} \mapsto v_{2}$. This isomorphism induces the permutation $\left(\begin{array}{lll}1 & 3 & 2\end{array}\right)$ on the triple $(1-a,-b, a+b)$. Therefore we may assume that $c \equiv-b(\bmod N)$.

The cycle structure at each vertex of $\Sigma$ is the following:

$$
\begin{aligned}
& v_{1}[i] \text { and } v_{6}[i] \text { : cycles of length } 2|\langle a+b\rangle| \text { and } 2|\langle-a+1\rangle|, \\
& v_{2}[i] \text { and } v_{5}[i] \text { : cycles of length } 2|\langle-a+1\rangle| \text { and } 2|\langle-b\rangle|, \\
& v_{3}[i] \text { and } v_{4}[i] \text { : cycles of length } 2|\langle a+b\rangle| \text { and } 2|\langle-b\rangle|,
\end{aligned}
$$

for all $i \in \mathbb{Z}_{N}$. Let $I$ be the union of $\langle b\rangle$ with a set of representatives for $\mathbb{Z}_{N} /\langle b\rangle$. Then $|I|=n+N / n-1$. We claim that $P=v_{1}[*] \cup v_{5}[I]$ is a plague. Using the sequence of pivots $v_{6}[I], v_{3}[I+b], v_{2}[I-1], v_{5}[(I+b) \cap I]$, and $v_{4}[(I+1) \cap$ $(I+b+1) \cap(I-b)]$, we see that $P$ spreads to

$v_{4}[I+1] \cup v_{2}[I+b] \cup v_{3}[I] \cup v_{6}[(I+1) \cap(I+b+1)] \cup v_{5}[(I+1) \cap(I+b+1) \cap(I-b)]$.

By looking at the evolution of $\chi_{P}$ at $v_{5}[*]$, Example 5.10 implies that $P$ spreads to $v_{5}[*]$. Since $P \cup v_{5}[*]$ is a plague, also $P$ is a plague. This implies the lemma. 
Lemma 7.25. Let $n \in \mathbb{N}$ with $2 \leq n \leq N / 2$. Then

$$
\frac{6 N}{n}+6 n-6 \leq 3 N+6 .
$$

Proof. The inequality is equivalent to $(n-2)(2 n-N) \leq 0$ and hence the claim follows.

Lemma 7.26. Let $\Sigma$ be a covering of $\Sigma_{6 D}$ with simply intersecting cycles. Then $\operatorname{imm}(\Sigma) \leq \omega(\Sigma)$.

Proof. There is an isomorphism $\Sigma_{6 D}^{N ; a, b} \rightarrow \Sigma_{6 D}^{N ; b+1, a-1}, v_{1} \mapsto v_{4}$ and this induces the permutation $(12)$ on $(1-a,-b, a+b)$. Using this and the isomorphism in the proof of Lemma 7.24, we may assume that

$$
0 \leq[1-a] \leq[-b] \leq[a+b]<N
$$

where for any $x \in \mathbb{Z}$ we let $[x] \in\{0,1, \ldots, N-1\}$ such that $[x] \equiv x(\bmod N)$.

Suppose first that $a=1$ and $b=0$. Then, using Lemma 7.24 with $c=1-a$, we obtain $\operatorname{imm}(\Sigma) \leq 1 / 3=\omega(\Sigma)$.

Assume now that $a \equiv 1(\bmod N), b \not \equiv 0(\bmod N)$ and $a+b \not \equiv 0(\bmod N)$. Then $N \geq 3$ by Lemma 7.22, Further, by (7.16)-(7.18),

$$
\omega(\Sigma)=\frac{(1 / 3) 4 N+(1 / 4) 2 N}{6 N}=\frac{11}{36} .
$$

Let $n=|\langle-b\rangle|$. Then $2 \leq c \leq N / 2$ by Lemma 7.22. Hence Lemma 7.24 for $c=-b$ and Lemma 7.25 imply that $\operatorname{imm}(\Sigma) \leq(9 N+6) / 36 N$ and hence $\operatorname{imm}(\Sigma) \leq \omega(\Sigma)$ since $N \geq 3$.

Finally we assume that $a \not \equiv 1(\bmod N), b \not \equiv 0(\bmod N)$, and $a+b \not \equiv 0$. Let $c \in\{1-a,-b, a+b\}$ and $n=|\langle c\rangle|$. Because of Lemma 7.22 we have $n \notin\{N, N / 2\}$ and hence $n \leq N / 3, N-3 n \geq 0$. Moreover, by choosing $c \in\{1-a,-b, a+b\}$ appropriately, we may assume that $n \geq 4$. Since all vertices of $\Sigma$ belong to cycles of length $\geq 4$, we obtain that $\omega(\Sigma)=1 / 4$. Then Lemma 7.24 implies that

$$
\operatorname{imm}(\Sigma) \leq \frac{N+N / n+n-1}{6 N}=\frac{3 N n-\left(N n-2 N-2 n^{2}+2 n\right)}{12 N n} \leq \frac{1}{4}
$$

because $N n-2 N-2 n^{2}+2 n=(N-2 n)(n-4)+2(N-3 n) \geq 0$.

\subsection{The graph $\Sigma_{7 A}$.}

Lemma 7.27. Let $\Sigma$ be a covering of $\Sigma_{7 A}$ in Figure 7.11 with simply intersecting cycles. Then $N=7$ and $(a, b, c)=(2,4,3)$.

Proof. The $x y$ cycles and their labels are: $\left(\begin{array}{llll}v_{1} & v_{3} & v_{6} & v_{2}\end{array}\right)$ with label $a-c+1$ and $\left(\begin{array}{lll}v_{5} & v_{4} & v_{7}\end{array}\right)$ with $b+c$. The $y x$-cycles are: $\left(v_{1} v_{2} v_{5} v_{4}\right)$ with $a-c+1$ and $\left(v_{6} v_{7} v_{3}\right)$ with $b+c$. Since $v_{1}$ and $v_{2}$ are on the same $x y$ - and $y x$-cycles, $N>1$ by Lemma 4.14. Lemma 4.13 on $v_{1}$ and $v_{7}$ implies that $3 a \equiv-1(\bmod N)$ and $2 b \equiv 1(\bmod N)$. By Lemma 4.16 on $v_{1}$ and $v_{7}$ we obtain $b \equiv-c(\bmod N)$ and $a \equiv c-1(\bmod N)$. From this the claim follows.

Lemma 7.28. Let $\Sigma$ be a covering of $\Sigma_{7 A}$ with simply intersecting cycles. Then $\operatorname{imm}(\Sigma) \leq \omega(\Sigma)$. 


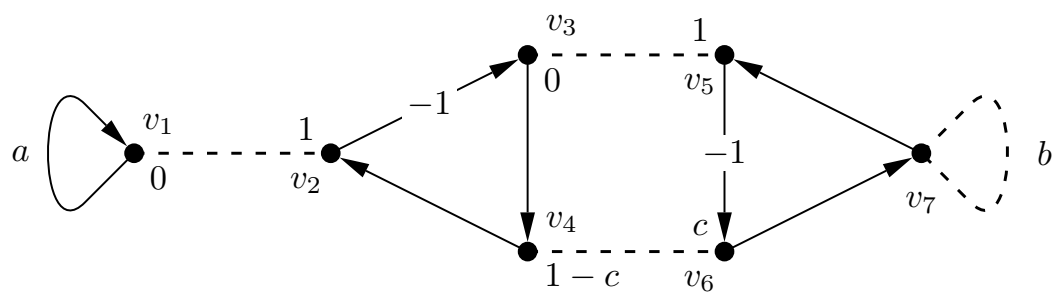

FIgURE 7.11. Schreier graph $\Sigma_{7 A}$ and its coverings

Proof. First we prove that $\operatorname{imm}(\Sigma) \leq 13 / 49$. By Lemma 7.27, $N=7$ and $(a, b, c)=$ $(2,4,3)$. Let $I=\{0, \ldots, 5\}$. We claim that $P=v_{1}[I] \cup v_{3}[*]$ is a plague of size 13. Use the pivots $v_{2}[I], v_{1}[(I-3) \cap(I+2)], v_{4}[(I-3) \cap(I+2)], v_{5}[I \cap(I-2)]$, $v_{3}[(I-3) \cap(I-1) \cap(I+2)]$ and $v_{7}[(I-2) \cap I \cap(I+1) \cap(I+3)]$ to let $P$ spread to

$$
\begin{aligned}
v_{4}[I] & \cup v_{2}[(I-2) \cap(I+3)] \cup v_{6}[(I-2) \cap I] \\
& \cup v_{7}[(I-2) \cap I] \cup v_{5}[\{0,1,3,5\}] \cup v_{5}[\{2,4,6\}] .
\end{aligned}
$$

Then $P$ spreads to $v_{5}[*]$. With $v_{3}[I-1]$ and with $v_{1}[3]$ as pivots, $P$ further spreads to $v_{2}[I] \cup v_{1}[6]$. Thus $P$ spreads to $v_{1}[*] \cup v_{3}[*]$. The above calculations with $I=\mathbb{Z}_{N}$ prove that $P$ is a plague. Hence $\operatorname{imm}(\Sigma) \leq 13 / 49$. By Lemma 7.27, the cycle structure of $\Sigma$ is

$$
\begin{aligned}
v_{1}[i] \text { and } v_{2}[i] & : \text { two } 4 \text {-cycles, } \\
v_{3}[i], v_{4}[i], v_{5}[i], v_{6}[i] & : \text { one } 3 \text {-cycle and one 4-cycle, } \\
v_{7}[i]: & \text { two } 3 \text {-cycles, }
\end{aligned}
$$

for all $i \in \mathbb{Z}_{N}$. Hence $\omega(\Sigma)=47 / 168>13 / 49 \geq \operatorname{imm}(\Sigma)$.

7.12. The graph $\Sigma_{8 A}$.

Lemma 7.29. Let $\Sigma$ be the covering of $\Sigma_{8 A}$ in Figure 7.12 with simply intersecting cycles. Then $\operatorname{imm}(\Sigma) \leq \omega(\Sigma)$.

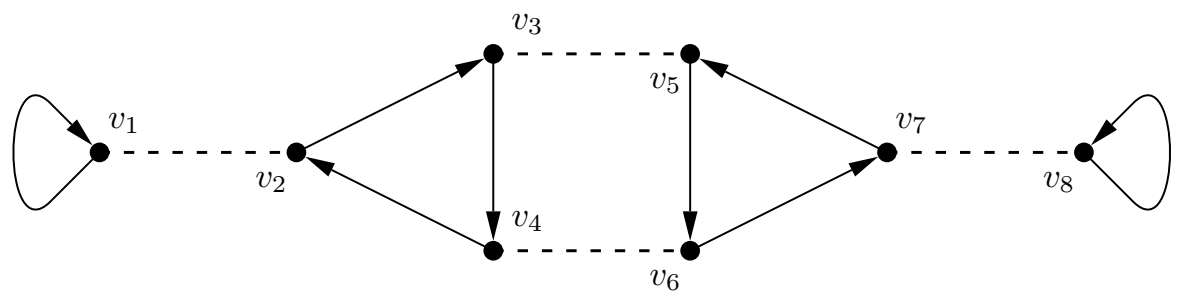

FiguRE 7.12. Schreier graph $\Sigma_{8 A}$ and its coverings 
Proof. A straightforward computation shows that $v_{1}[*] \cup v_{3}[*]$ is a plague of size $2 N$ and hence $\operatorname{imm}(\Sigma) \leq 1 / 4$. Since all cycles have length $\geq 4$, the claim follows.

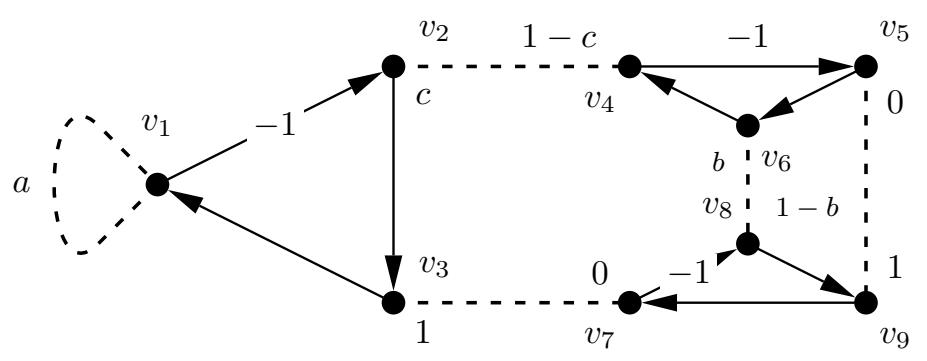

FigURE 7.13. Schreier graph $\Sigma_{9 A}$ and its coverings

\subsection{The graph $\Sigma_{9 A}$.}

Lemma 7.30. Let $\Sigma$ be a covering of $\Sigma_{9 A}$ in Figure 7.13 with simply intersecting cycles. Then $N>1,2 a \equiv 1(\bmod N), N$ is odd, $c \equiv a+1(\bmod N)$ and $\langle b-1\rangle \cap$ $\langle a+b\rangle=0$.

Proof. The $x y$-cycles and their labels are: $\left(v_{1} v_{2} v_{5} v_{7}\right)$ with label $a-c+1,\left(v_{6} v_{9}\right)$ with $-b+1$ and $\left(v_{3} v_{8} v_{4}\right)$ with $b+c-1$. The $y x$-cycles are: $\left(\begin{array}{llll}v_{1} & v_{4} & v_{9} & v_{3}\end{array}\right)$ with $a-c+1,\left(v_{2} v_{7} v_{6}\right)$ with $b+c-1$ and $\left(v_{5} v_{8}\right)$ with $-b+1$. Lemmas 4.13 and 4.16 on $v_{1}$ imply $2 a \equiv 1(\bmod N)$ and $c \equiv a+1(\bmod N)$. Since $v_{2}$ and $v_{7}$ are on the same $x y$-cycle and the same $y x$-cycle, we conclude that $N>1$ by Lemma 4.14. By Lemma 4.15 on $v_{6}$ the claim follows.

Lemma 7.31. Let $\Sigma$ be a covering of $\Sigma_{9 A}$ with simply intersecting cycles. Then

$$
\operatorname{imm}(\Sigma) \leq \begin{cases}8 / 27 & \text { if } a+b \equiv 0(\bmod N) \text { and } b \equiv 1(\bmod N), \\ 11 / 45 & \text { if } a+b \neq \equiv(\bmod N) \text { and } b \neq \equiv 1(\bmod N), \\ 7 / 27 & \text { otherwise. }\end{cases}
$$

Proof. There are four cases to consider. Assume first that $b \equiv 1(\bmod N)$ and $a+b \equiv 0(\bmod N)$. Lemma 7.30 implies then that $N=3, a \equiv 2(\bmod N), b \equiv$ $1(\bmod N)$, and $c \equiv 0(\bmod N)$. Let $I=\{0,1\}$. Then $P=v_{1}[I] \cup v_{2}[*] \cup v_{5}[*]$ is a plague of size 8 . Indeed,

$$
\begin{array}{c|cccccc}
\text { pivot } & v_{4}[*] & v_{1}[I+1] & v_{3}[I-1] & v_{8}[I+1] & v_{2}[I \cap(I-1)] & v_{5}[I+1] \\
\hline & v_{6}[*] & v_{3}[I+1] & v_{7}[I-1] & v_{9}[I+2] & v_{4}[I \cap(I+1)] & v_{4}[I+2]
\end{array}
$$

and hence, since $(I \cap(I+1)) \cup(I+2)=\mathbb{Z}_{N}, P$ spreads to $v_{4}[*]$. From this the claim follows.

Assume now that $b \not \equiv 1(\bmod N)$ and that $a+b \equiv 0(\bmod N)$. Then $|\langle b-1\rangle| \geq 3$ because $N$ is odd. Let $I$ be a set of representatives for $\mathbb{Z}_{N} /\langle b-1\rangle$. We claim that $v_{1}[*] \cup v_{2}[*] \cup v_{5}[I]$ is a plague. We compute

\begin{tabular}{c|ccccccc} 
pivot & $v_{1}[*]$ & $v_{3}[*]$ & $v_{2}[*]$ & $v_{4}[I]$ & $v_{5}[I-1]$ & $v_{7}[I]$ & $v_{6}[I+b-1]$ \\
\hline & $v_{3}[*]$ & $v_{7}[*]$ & $v_{4}[*]$ & $v_{6}[I]$ & $v_{9}[I]$ & $v_{8}[I]$ & $v_{5}[I+b-1]$
\end{tabular}


and the claim follows from Example 5.9. Therefore

$$
\operatorname{imm}(\Sigma) \leq \frac{2 N+|I|}{9 N} \leq 7 / 27
$$

since $|I| \leq N / 3$.

Assume now that $b \equiv 1(\bmod N)$ and $a+b \not \equiv 0(\bmod N)$. Let $I$ be a set of representatives for $\mathbb{Z}_{N} /\langle a+b\rangle$. Then $P=v_{4}[*] \cup v_{8}[*] \cup v_{2}[I]$ is a plague. Indeed, $P$ spreads according to the following table:

$$
\begin{array}{c|ccccc}
\text { pivot } & v_{6}[*] & v_{9}[*] & v_{4}[I-a-1] & v_{8}[I-a-b-1] & v_{7}[I-a-b] \\
\hline & v_{5}[*] & v_{7}[*] & v_{6}[I-a-1] & v_{9}[I-a-b] & v_{3}[I-a-b+1]
\end{array}
$$

Pivoting from $v_{2}[I-a-b]$ and $v_{3}[I-a-b], P$ spreads further to $v_{1}[I-a-b+$ 1] $\cup v_{2}[I-a-b]$. As before, since $|I| \leq N / 3$ and using Example 5.9 with $\lambda=a+b$, we conclude that $P$ spreads to $v_{2}[*]$. Hence $P$ is a plague and $\operatorname{imm}(\Sigma) \leq 7 / 27$.

Finally, assume that $b \not \equiv 1(\bmod N)$ and $a+b \not \equiv 0(\bmod N)$. From Lemma 7.30 we obtain that $N$ is odd, $\langle a+b\rangle \cap\langle b-1\rangle=0$, and $c \equiv a+1(\bmod N)$. If $|\langle a+b\rangle|=3$, then $|\langle b-1\rangle| \geq 5$ and $v_{1}[*] \cup v_{2}[*] \cup v_{5}[I]$ is a plague, where $I$ is a set of representatives for $\mathbb{Z}_{N} /\langle b-1\rangle$. Indeed, the calculations

$$
\begin{array}{c|ccccccc}
\text { pivot } & v_{1}[*] & v_{2}[*] & v_{3}[*] & v_{4}[I] & v_{5}[I-1] & v_{8}[I-1] & v_{4}[I+b-1] \\
\hline & v_{3}[*] & v_{4}[*] & v_{7}[*] & v_{6}[I] & v_{9}[I] & v_{6}[I+b-1] & v_{5}[I+b-1]
\end{array}
$$

together with Example 5.9 with $\lambda=1-b$ show that $P$ is a plague if $P \cup v_{5}[*]$ is a plague. However, the latter is easy to check. On the other hand, if $|\langle a+b\rangle| \geq 5$, then let $P=v_{4}[*] \cup v_{8}[*] \cup v_{2}[I]$, where $I$ is a set of representatives for $\mathbb{Z}_{N} /\langle a+b\rangle$. Then $P$ is a plague. Indeed, the calculations

\begin{tabular}{c|ccccc} 
pivot & $v_{6}[*]$ & $v_{9}[*]$ & $v_{4}[I-a-1]$ & $v_{8}[I-a-b-1]$ & $v_{7}[I-a-b]$ \\
\hline & $v_{5}[*]$ & $v_{7}[*]$ & $v_{6}[I-a-1]$ & $v_{9}[I-a-b]$ & $v_{3}[I-a-b+1]$ \\
pivot & & & & $v_{2}[I-a-b]$ & $v_{3}[I-a-b]$ \\
\hline & & & & $v_{1}[I-a-b+1]$ & $v_{2}[I-a-b]$
\end{tabular}

together with Example 5.9 with $\lambda=a+b$ show that $P$ is a plague if $P \cup v_{2}[*]$ is a plague. Again, the latter is easy to check. Since $|I| \leq N / 5$, in the last two cases we obtain that

$$
\operatorname{imm}(\Sigma) \leq \frac{2 N+|I|}{9 N} \leq \frac{2 N+N / 5}{9 N}=11 / 45
$$

This completes the proof.

Lemma 7.32. Let $\Sigma$ be a covering of $\Sigma_{9 A}$ with simply intersecting cycles. Then $\operatorname{imm}(\Sigma) \leq \omega(\Sigma)$.

Proof. The cycle structure at each vertex of $\Sigma$ is the following:

$v_{1}[i]$ : two 4-cycles, $v_{2}[i], v_{3}[i], v_{4}[i], v_{7}[i]:$ one 4 -cycle and one cycle of length $3|\langle a+b\rangle|$, $v_{5}[i], v_{9}[i]:$ one 4 -cycle and one cycle of length $2|\langle b-1\rangle|$, $v_{6}[i], v_{8}[i]$ : cycles of length $3|\langle a+b\rangle|$ and $2|\langle b-1\rangle|$, 
for all $i \in \mathbb{Z}_{N}$. From (7.20)-(7.23) it is straightforward to compute the weight at every vertex of $\Sigma$ and $\omega(\Sigma)$ :

$$
\omega(\Sigma)= \begin{cases}11 / 36 & \text { if } a \equiv-b(\bmod N), b \equiv 1(\bmod N), \\ 5 / 18 & \text { if } a \equiv-b(\bmod N), b \neq 1(\bmod N), \\ 31 / 108 & \text { if } a \not \equiv-b(\bmod N), b \equiv 1(\bmod N), \\ 1 / 4 & \text { if } a \neq \equiv-b(\bmod N), b \neq \equiv 1(\bmod N) .\end{cases}
$$

From this and from Lemma 7.31 the claim follows.

\subsection{The graph $\Sigma_{12 A}$.}

Lemma 7.33. Let $\Sigma$ be a covering of $\Sigma_{12 A}$ in Figure 7.14 with simply intersecting cycles. Then $\operatorname{imm}(\Sigma) \leq 1 / 4=\omega(\Sigma)$.

Proof. In $\Sigma$, all cycles have length $\geq 4$; hence $\omega(\Sigma)=1 / 4$. A straightforward computation shows that $v_{1}[*] \cup v_{2}[*] \cup v_{5}[*]$ is a plague of $\Sigma$ of size $3 N$ and hence $\operatorname{imm}(\Sigma) \leq 1 / 4=\omega(\Sigma)$.

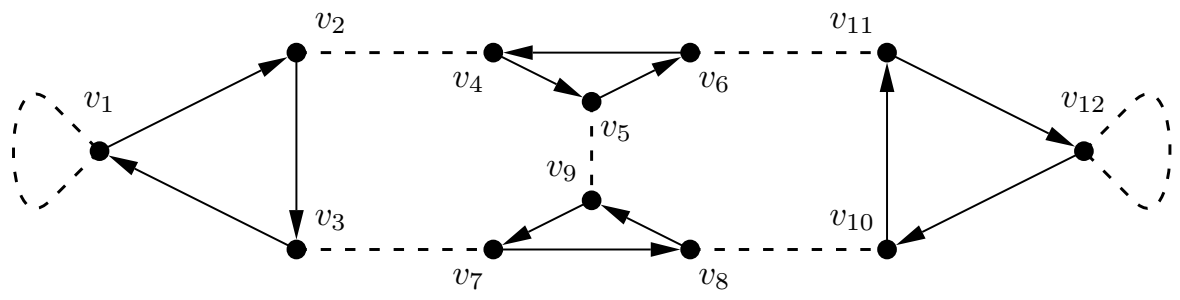

FiguRE 7.14. Schreier graph $\Sigma_{12 A}$

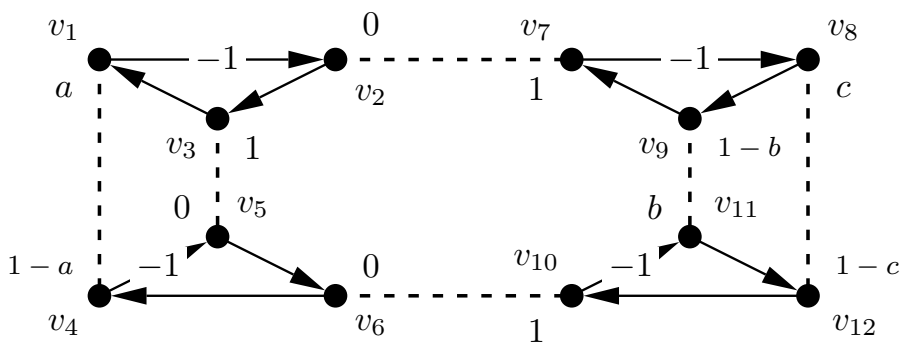

FiguRE 7.15. Schreier graph $\Sigma_{12 B}$ and its coverings

\subsection{The graph $\Sigma_{12 B}$.}

Lemma 7.34. Let $\Sigma$ be a covering of $\Sigma_{12 B}$ in Figure 7.15] with simply intersecting cycles. Then $N>1$ and the following conditions are satisfied:

(1) $\langle 1-a\rangle \cap\langle a-c\rangle=0$,

(2) $\langle 1-b\rangle \cap\langle a-c\rangle=0$,

(3) $\langle 1-b\rangle \cap\langle 1-a\rangle=0$,

(4) $\langle 1-b\rangle \cap\langle b+c\rangle=0$,

(5) $\langle a-c\rangle \cap\langle b+c\rangle=0$,

(6) $(-c+\langle a-c\rangle) \cap\langle b-1\rangle=\emptyset$. 
Proof. The yx-cycles and their labels are: $\left(v_{3} v_{4}\right)$ with $1-a,\left(v_{8} v_{11}\right)$ with $b+c$, $\left(\begin{array}{llll}v_{1} & v_{7} & v_{12} & v_{6}\end{array}\right)$ with $a-c$ and $\left(\begin{array}{llll}v_{2} & v_{5} & v_{10} & v_{9}\end{array}\right)$ with $1-b$. The $x y$-cycles are: $\left(v_{1} v_{5}\right)$ with $1-a,\left(\begin{array}{ll}v_{9} & v_{12}\end{array}\right)$ with $b+c,\left(\begin{array}{llll}v_{2} & v_{8} & v_{10} & v_{4}\end{array}\right)$ with $a-c$ and $\left(\begin{array}{llll}v_{3} & v_{6} & v_{11} & v_{7}\end{array}\right)$ with $1-b$. Then Lemma 4.15 implies (11)-(5). Moreover, Lemma 4.14 on $v_{6}$ and $v_{7}$ implies that $N>1$. Finally, the claim (6) follows from Lemma 4.17 on $v_{2}$ and $v_{10}$. Indeed, the $x y$-path starting in $v_{2}[i]$ for some $i \in \mathbb{Z}_{N}$, which has length $4 k+2$ with $k \in \mathbb{Z}$, ends in $v_{10}[1-c+k(a-c)]$, and any $y x$-path of length $4 l+2$ with the same starting point ends in $v_{10}[1+l(1-b)]$.

Lemma 7.35. Let $\Sigma$ be a covering of $\Sigma_{12 B}$ as in Figure 7.15 with simply intersecting cycles. Then

$$
\operatorname{imm}(\Sigma) \leq \begin{cases}\frac{3 N+1}{12 N} & \text { if } a \equiv 1(\bmod N) \text { or } b+c \equiv 0(\bmod N), \\ 1 / 4 & \text { otherwise. }\end{cases}
$$

Proof. The cycle structure at each vertex of $\Sigma$ is:

$$
\begin{array}{r}
v_{1}[i], v_{4}[i]: \text { cycles of length } 4|\langle a-c\rangle| \text { and } 2|\langle 1-a\rangle|, \\
v_{2}[i], v_{6}[i], v_{7}[i], v_{10}[i]: \text { cycles of length } 4|\langle 1-b\rangle| \text { and } 4|\langle a-c\rangle|, \\
v_{3}[i], v_{5}[i]: \text { cycles of length } 2|\langle 1-a\rangle| \text { and } 4|\langle 1-b\rangle|, \\
v_{8}[i], v_{12}[i]: \text { cycles of length } 2|\langle b+c\rangle| \text { and } 4|\langle a-c\rangle|, \\
v_{9}[i], v_{11}[i]: \text { cycles of length } 2|\langle b+c\rangle| \text { and } 4|\langle 1-b\rangle|,
\end{array}
$$

for all $i \in \mathbb{Z}_{N}$.

Assume first that $a \equiv 1(\bmod N)$ or $b+c \equiv 0(\bmod N)$. We claim that there exists a subset $I \subseteq \mathbb{Z}_{N}$ of size one such that the set $P=v_{2}[*] \cup v_{5}[*] \cup v_{9}[*] \cup v_{10}[I]$ is a plague of size $3 N+1$. We compute

$$
\begin{array}{c|cccccc}
\text { pivot } & v_{3}[*] & v_{4}[*] & v_{7}[*] & v_{11}[I-1] & v_{10}[I] & v_{12}[I] \\
\hline & v_{1}[*] & v_{6}[*] & v_{8}[*] & v_{12}[I] & v_{11}[I] & v_{10}[I+1]
\end{array}
$$

and hence $P$ spreads to $v_{10}[*]$ by Example 5.9 . Thus $P$ is a plague since $P \cup v_{10}[*]$ is a plague. In this case $\operatorname{imm}(\Sigma) \leq(3 N+1) / 12 N$.

Assume now that $a \not \equiv 1(\bmod N)$ and $b+c \not \equiv 0(\bmod N)$. Let $I$ be a set of representatives for $\mathbb{Z}_{N} /\langle a-1\rangle$ and let $J$ be a set of representatives for $\mathbb{Z}_{N} /\langle b+c\rangle$. We claim that $v_{3}[I] \cup v_{8}[J] \cup v_{1}[*] \cup v_{2}[*]$ is a plague. We compute

$$
\begin{array}{c|cccc}
\text { pivot } & v_{3}[*] & v_{4}[*] & v_{5}[I-1] & v_{1}[I+a-1] \\
\hline & v_{5}[*] & v_{6}[*] & v_{4}[I] & v_{3}[I+a-1]
\end{array}
$$

and therefore $P$ spreads to $v_{3}[*] \cup v_{4}[*]$ by Example [5.9] Now the calculations

$$
\begin{array}{c|cccc}
\text { pivot } & v_{2}[*] & v_{6}[*] & v_{9}[J] & v_{12}[J+b] \\
\hline & v_{7}[*] & v_{10}[*] & v_{11}[J+b] & v_{8}[I+b+c]
\end{array}
$$

show that $P$ spreads to $v_{8}[*]$ by Example 5.9, Then the claim follows, and in this case $\operatorname{imm}(\Sigma) \leq 1 / 4$ since $|I|+|J| \leq N$.

Lemma 7.36. Let $\Sigma$ be a covering of $\Sigma_{12 B}$ with simply intersecting cycles. Then $\operatorname{imm}(\Sigma) \leq \omega(\Sigma)$. 
Proof. Using (7.24)-(7.28) we obtain

$$
\omega(\Sigma)= \begin{cases}11 / 36 & \text { if } a \equiv 1(\bmod N) \text { and } b+c \equiv 0(\bmod N), \\ 5 / 18 & \text { if } a \equiv 1(\bmod N) \text { and } b+c \neq \equiv 0(\bmod N), \\ 5 / 18 & \text { if } a \neq 1(\bmod N) \text { and } b+c \equiv 0(\bmod N), \\ 1 / 4 & \text { otherwise. }\end{cases}
$$

First notice that if $N=2$, then $a \equiv 1(\bmod N), b \equiv 1(\bmod N)$, and $c \equiv 1(\bmod N)$ by Lemma 7.34(6). Then $\operatorname{imm}(\Sigma) \leq \frac{3 N+1}{12 N}=7 / 24<11 / 36=\omega(\Sigma)$ by Lemma 7.35. and the claim holds. So we may assume that $N>2$.

If $a \equiv 1(\bmod N)$ or $b+c \equiv 0(\bmod N)$, then Lemma 7.35 and $N \geq 3$ imply that

$$
\operatorname{imm}(\Sigma) \leq \frac{3 N+1}{12 N} \leq 10 / 36 \leq \omega(\Sigma) .
$$

Otherwise $\operatorname{imm}(\Sigma) \leq 1 / 4=\omega(\Sigma)$ by Lemma 7.35 ,

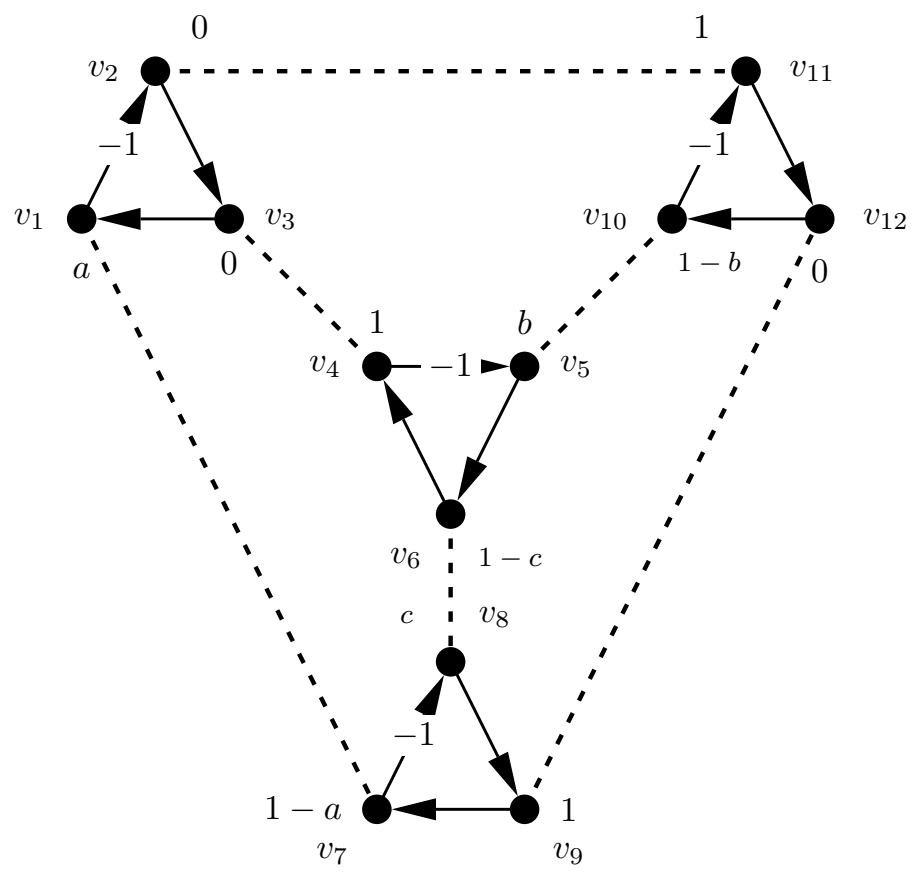

FIGURE 7.16. Schreier graph $\Sigma_{12 C}$ and its coverings

\subsection{The graph $\Sigma_{12 C}$.}

Lemma 7.37. Let $\Sigma$ be a covering of $\Sigma_{12 C}$ in Figure 7.16 with simply intersecting cycles. Then the following hold:
(1) $\langle-a-c+1\rangle \cap\langle a+1\rangle=0$,
(2) $\langle a+1\rangle \cap\langle-b\rangle=0$,
(3) $\langle-b\rangle \cap\langle-a-c+1\rangle=0$,
(4) $\langle-b\rangle \cap\langle b+c\rangle=0$,
(5) $\langle b+c\rangle \cap\langle-a-c+1\rangle=0$,
(6) $\langle b+c\rangle \cap\langle a+1\rangle=0$. 
Proof. The yx-cycles and their labels are: $\left(v_{1} v_{11} v_{9}\right)$ with label $a+1,\left(v_{2} v_{4} v_{10}\right)$ with $-b,\left(\begin{array}{lll}v_{3} & v_{7} & v_{6}\end{array}\right)$ with $-a-c+1$ and $\left(v_{5} v_{8} v_{12}\right)$ with $b+c$. The $x y$-cycles are: $\left(\begin{array}{lll}v_{1} & v_{8} & v_{4}\end{array}\right)$ with $-a-c+1,\left(\begin{array}{lll}v_{2} & v_{12} & v_{7}\end{array}\right)$ with $a+1,\left(\begin{array}{lll}v_{11} & v_{3} & v_{5}\end{array}\right)$ with $-b$ and $\left(\begin{array}{lll}v_{9} & v_{10} & v_{6}\end{array}\right)$ with $b+c$. Then the claim follows from Lemma 4.15]

Example 7.38. The trivial covering of $\Sigma_{12 C}$ is isomorphic to the Hurwitz orbit of size 12 of [6. Figure 12]. The covering $\Sigma_{12 C}^{2 ; 1,0,0}$ is isomorphic to the Hurwitz orbit of size 24 of [6, Figure 14].

Lemma 7.39. Assume that $N / n \geq 6$ and $n \geq 5$. Then

$$
n \frac{N / n+1}{2}+\frac{N / n-1}{2}+\frac{N}{n} \leq N \text {. }
$$

Proof. After multiplication with $2 n$ the inequality takes the form $N n-3 N+n-$ $2 n^{2} \geq 0$ or $(N-6 n)(n-3)+4 n(n-5)+3 n \geq 0$.

Lemma 7.40. Let $\Sigma$ be a covering of $\Sigma_{12 C}$ in Figure 7.16 with simply intersecting cycles. Then

$$
\operatorname{imm}(\Sigma) \leq \begin{cases}1 / 3 & \text { if } \Sigma \text { is the trivial covering, } \\ 7 / 24 & \text { if } \Sigma=\Sigma_{12 C}^{2 ; 1,0,0} \\ 7 / 24 & \text { if } 1+a \equiv 1-a-c \equiv b \equiv 0(\bmod N), \\ & b+c \neq \equiv(\bmod N), \\ 5 / 18 & \text { if } 1+a \equiv 1-a-c \equiv 0(\bmod N), \\ & b, b+c \neq 0(\bmod N), \\ 1 / 4 & \text { otherwise. }\end{cases}
$$

Proof. The cycle structure at each vertex is the following:

$$
\begin{gathered}
v_{1}[i], v_{7}[i]: \text { cycles of length } 3|\langle 1-a-c\rangle| \text { and } 3|\langle 1+a\rangle|, \\
v_{2}[i], v_{11}[i]: \text { cycles of length } 3|\langle 1+a\rangle| \text { and } 3|\langle-b\rangle|, \\
v_{3}[i], v_{4}[i]: \text { cycles of length } 3|\langle-b\rangle| \text { and } 3|\langle 1-a-c\rangle|, \\
v_{5}[i], v_{10}[i]: \text { cycles of length } 3|\langle b+c\rangle| \text { and } 3|\langle-b\rangle|, \\
v_{6}[i], v_{8}[i]: \text { cycles of length } 3|\langle b+c\rangle| \text { and } 3|\langle 1-a-c\rangle|, \\
v_{9}[i], v_{12}[i]: \text { cycles of length } 3|\langle b+c\rangle| \text { and } 3|\langle 1+a\rangle|,
\end{gathered}
$$

for all $i \in \mathbb{Z}_{N}$. Without loss of generality we may assume that

$$
|\langle 1+a\rangle| \leq|\langle 1-a-c\rangle| \leq|\langle-b\rangle| \leq|\langle b+c\rangle| \text {. }
$$

We split the proof into five cases according to the number of trivial groups in (7.35). However, two of these cases will be considered simultaneously.

First assume that $1+a \equiv 1-a-c \equiv b \equiv b+c \equiv 0(\bmod N)$. Then $N=1$ (the trivial covering) or $N=2$ and $(a, b, c)=(1,0,0)$. Since these orbits appear as Hurwitz orbits of braided racks (see Example 7.38), the claim follows from 6, Proposition 10].

Assume now that $1+a \equiv a+c-1 \equiv b \equiv 0(\bmod N)$ and $b+c \not \equiv 0(\bmod N)$. Then $a \equiv-1(\bmod N), b \equiv 0(\bmod N), c \equiv 2(\bmod N)$, and $N \geq 3$. Let $I$ be a set of representatives for $\mathbb{Z}_{N} /\langle b+c\rangle$. Then $|I| \leq N / 2$. We claim that $P=v_{1}[*] \cup v_{2}[*] \cup v_{3}[*] \cup v_{5}[I]$ is a plague. With the sequence of pivots $v_{1}[*]$, 
$v_{2}[*], v_{3}[*], v_{4}[I], v_{5}[I-1], v_{6}[I], v_{7}[I+c], v_{9}[I+c], v_{10}[I+c]$ we see that $P$ spreads to

$v_{7}[*] \cup v_{11}[*] \cup v_{4}[*] \cup v_{6}[I] \cup v_{10}[I-b] \cup v_{8}[I+c] \cup v_{9}[I+c] \cup v_{12}[I+c] \cup v_{5}[I+b+c]$.

Hence the claim follows from Example 5.9 for the fiber over $v_{5}$.

Now assume that exactly two groups of (7.35) are trivial, i.e., $1+a \equiv a+c-1 \equiv$ $0(\bmod N)$, and $b, b+c \not \equiv 0(\bmod N)$. Then $a \equiv-1(\bmod N), c \equiv 2(\bmod N)$, and $1<|\langle b\rangle| \leq|\langle b+c\rangle|$. From Lemma 7.34 we obtain $\langle b\rangle \cap\langle b+c\rangle=0$ and hence $|\langle b+c\rangle| \geq 3$. Therefore the previous computation with $|I| \leq N / 3$ yields the claim.

Finally, assume that $1-a-c, b, b+c \not \equiv 0(\bmod N)$. We prove that $\operatorname{imm}(\Sigma) \leq 1 / 4$. Let $n=|\langle b+c\rangle|$. Instead of $|\langle 1-a-c\rangle| \leq|\langle b\rangle| \leq|\langle b+c\rangle|$ we assume that $|\langle 1-a-c\rangle|,|\langle b\rangle| \leq n$ and $n|\langle 1-a-c\rangle| \neq N$. This is possible since any subgroup of $\mathbb{Z}_{N}$ is uniquely determined by its order and any two of these three subgroups have trivial intersection by Lemma 7.37 Observe that $n \geq 5$. We claim that there exist two subsets $I, J \subseteq \mathbb{Z}_{N}$ with $|I|+|J| \leq N$, such that

$$
P=v_{1}[*] \cup v_{2}[*] \cup v_{3}[I] \cup v_{5}[J]
$$

is a plague. We split the proof into several steps.

Step 1. The following computation shows that if $x+a+c \in I$ and $x+b+c \in I \cap J$, then $P$ spreads to $\left\{v_{5}[x]\right\}$ :

\begin{tabular}{c|c} 
pivot & \\
\hline$v_{3}[*]$ & $v_{4}[*]$ \\
$v_{1}[I]$ & $v_{7}[I-a+1]$ \\
$v_{4}[I \cap J]$ & $v_{6}[I \cap J]$ \\
$v_{5}[(I-1) \cap(J-1)]$ & $v_{10}[(I-b) \cap(J-b)]$ \\
$v_{11}[(I-b-1) \cap(J-b-1)]$ & $v_{12}[(I-b) \cap(J-b)]$ \\
$v_{9}[(I-a) \cap(I-b) \cap(J-b)]$ & $v_{8}[(I-a) \cap(I-b) \cap(J-b)]$ \\
$v_{6}[(I-a-c) \cap(I-b-c) \cap(J-b-c)]$ & $v_{5}[(I-a-c) \cap(I-b-c) \cap(J-b-c)]$
\end{tabular}

Step 2. We claim that if $x$ satisfies $x+a+c-1 \in I, x+a+c \in I$ and $x+b+c \in I \cap J$, then $P$ spreads to $\left\{v_{3}[x], v_{5}[x]\right\}$. This follows from Step 1 and the following fact: if $x+a+c-1 \in I$ and $x \in J$, then $P$ spreads to $\left\{v_{3}[I]\right\}$. The proof is obtained from the following table:

\begin{tabular}{c|c} 
pivot & \\
\hline$v_{3}[*]$ & $v_{4}[*]$ \\
$v_{6}[J]$ & $v_{8}[J+c]$ \\
$v_{7}[J+c]$ & $v_{9}[J+c]$ \\
$v_{1}[I]$ & $v_{7}[I+1-a]$ \\
$v_{8}[(I-a) \cap(J+c-1)]$ & $v_{6}[(I-a-c+1) \cap J]$ \\
$v_{4}[(I+1-a-c) \cap J]$ & $v_{3}[(I-a-c+1) \cap J]$
\end{tabular}

Step 3. Since $\langle a+c-1\rangle \cap\langle b+c\rangle=0$ and $\langle b+c\rangle=\langle N / n\rangle$, it follows that $a+c-1 \not \equiv 0(\bmod N / n)$. The order of $a+c-1 \operatorname{modulo} N / n$ is the same as modulo $N$, as $\langle a+c-1\rangle \cap\langle b+c\rangle=0$. Since $n|\langle a+c-1\rangle| \neq N$, we obtain that $a+c-1 \not \equiv-1(\bmod N / n)$. Using the prime factorization of $N$ and three non-zero parameters, from the conditions on the parameters we also get that $N / n \geq 6$. (The generators of the groups generated by the non-zero parameters have at least two distinct prime factors.) 
Step 4 . Let $J$ be a set of representatives for $\mathbb{Z}_{N} /\langle b+c\rangle$. Let

$$
I=J \cup\{i+\langle b+c\rangle \mid 0 \leq i \leq(N / n-1) / 2\} .
$$

Then $|J|=N / n$ and $|I| \leq n \frac{N / n+1}{2}+\frac{N / n-1}{2}$. By Lemma 7.39, $|I|+|J| \leq N$. So it is enough to show that (7.36) is a plague. For that purpose, we use Example 5.11 with $m=N / n$ and $\lambda=1-a-c$.

Lemma 7.41. Let $\Sigma$ be a covering of $\Sigma_{12 C}$ with simply intersecting cycles. Then $\operatorname{imm}(\Sigma) \leq \omega(\Sigma)$.

Proof. As in the proof of Lemma 7.40, without loss of generality we may assume that $|\langle 1+a\rangle| \leq|\langle 1-a-c\rangle| \leq|\langle-b\rangle| \leq|\langle b+c\rangle|$. A straightforward computation shows that

$$
(7.37) \omega(\Sigma)= \begin{cases}1 / 3 & \text { if } \Sigma \text { is the trivial covering of } \Sigma_{12 C}, \\ 7 / 24 & \text { if } 1+a, 1-a-c, b, b+c \equiv 0(\bmod N), N=2, \\ 7 / 24 & \text { if } 1+a, 1-a-c, b \equiv 0(\bmod N), b+c \neq \equiv 0(\bmod N), \\ 41 / 144 & \text { if } 1+a, 1-a-c \equiv 0(\bmod N), b, b+c \not \equiv 0(\bmod N), \\ 13 / 48 & \text { if } 1+a \equiv 0(\bmod N), 1-a-c, b, b+c \not \equiv 0(\bmod N), \\ 1 / 4 & \text { if } 1+a, 1-a-c, b, b+c \not \equiv 0(\bmod N) .\end{cases}
$$

Hence the claim follows from Lemma 7.40

Lemma 7.42. Let $X$ be an injective indecomposable rack. Assume that $X$ has at least one Hurwitz orbit $\mathcal{O}$ isomorphic to the covering $\Sigma_{12 C}^{1 ; 0,0,0}$. Then $x \triangleright(x \triangleright y)=z$ for all $(x, y, z) \in \mathcal{O}$.

Proof. See the proof of [6, Proposition 9].

\subsection{The graph $\Sigma_{18 A}$.}

Lemma 7.43. Let $\Sigma$ be a covering of $\Sigma_{18 A}$ in Figure 7.17 with simply intersecting cycles. Then
(1) $\langle-a+c+d+1\rangle \cap\langle a+1\rangle=0$,
(2) $\langle-a+c+d+1\rangle \cap\langle-c+1\rangle=0$,
(3) $\langle-a+c+d+1\rangle \cap\langle-b-d\rangle=0$,
(4) $\langle-a+c+d+1\rangle \cap\langle b\rangle=0$,
(5) $\langle a+1\rangle \cap\langle-c+1\rangle=0$,
(6) $\langle a+1\rangle \cap\langle-b-d\rangle=0$,
(7) $\langle a+1\rangle \cap\langle b\rangle=0$,
(8) $\langle-c+1\rangle \cap\langle-b-d\rangle=0$,
(9) $\langle-b-d\rangle \cap\langle b\rangle=0$.

Proof. The $x y$-cycles and their labels are: $\left(\begin{array}{llll}v_{1} & v_{14} & v_{10} & v_{7}\end{array}\right)$ with $-a+c+d+1$, $\left(\begin{array}{llll}v_{2} & v_{5} & v_{18} & v_{13}\end{array}\right)$ with $1+a,\left(\begin{array}{lll}v_{3} & v_{8} & v_{4}\end{array}\right)$ with $1-c,\left(\begin{array}{llll}v_{6} & v_{9} & v_{11} & v_{17}\end{array}\right)$ with $-b-d$ and $\left(\begin{array}{lll}v_{12} & v_{15} & v_{16}\end{array}\right)$ with $b$. The $y x$-cycles are: $\left(v_{1} v_{4} v_{17} v_{15}\right)$ with $1+a,\left(v_{2} v_{7} v_{6}\right)$ with $1-c,\left(\begin{array}{llll}v_{3} & v_{13} & v_{12} & v_{9}\end{array}\right)$ with $-a+c+d+1,\left(\begin{array}{llll}v_{5} & v_{8} & v_{10} & v_{16}\end{array}\right)$ with $-b-d$ and $\left(\begin{array}{lll}v_{11} & v_{14} & v_{18}\end{array}\right)$ with $b$. Lemma 4.15 implies the claim.

Lemma 7.44. Let $\Sigma$ be a covering of $\Sigma_{18 A}$ with simply intersecting cycles. Then $\operatorname{imm}(\Sigma) \leq \omega(\Sigma)$. 


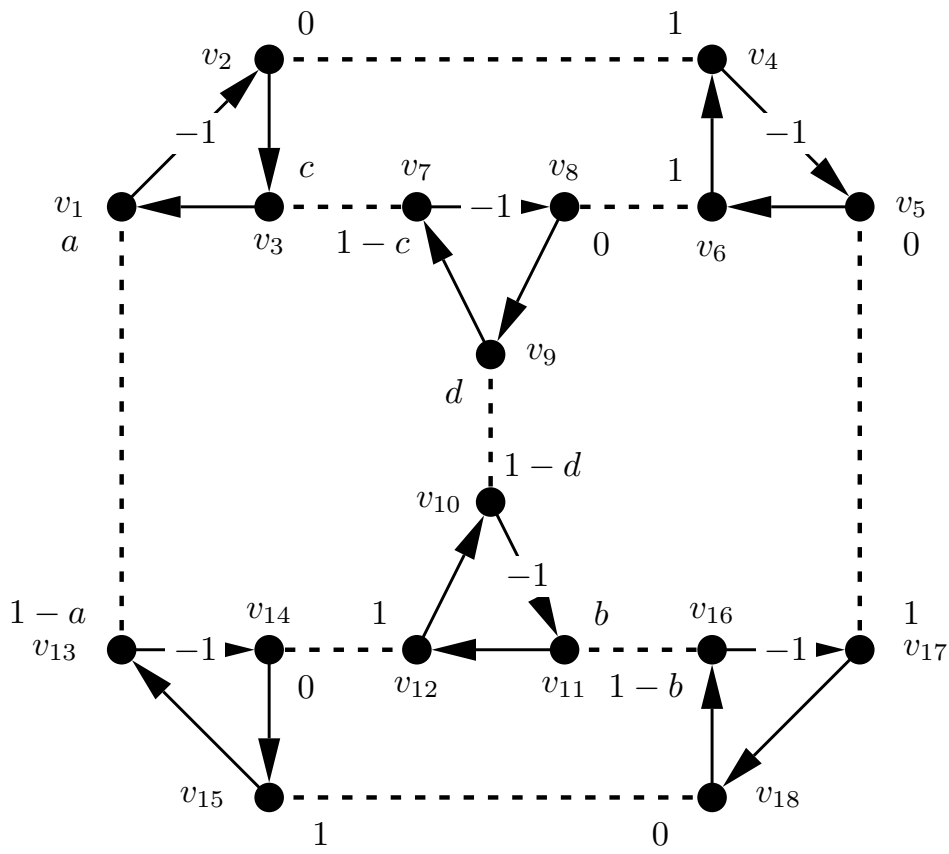

FiguRE 7.17. Schreier graph $\Sigma_{18 A}$

Proof. Let $I$ be a set of representatives for $\mathbb{Z}_{N} /\langle b\rangle$. We claim that

$$
P=v_{1}[*] \cup v_{2}[*] \cup v_{3}[*] \cup v_{5}[*] \cup v_{11}[I]
$$

is a plague. With the sequence of pivots $v_{1}[*], v_{3}[*], v_{4}[*], v_{8}[*], v_{7}[*], v_{6}[*], v_{9}[*]$ and $v_{5}[*]$ we obtain that $P$ spreads to

$$
v_{13}[*] \cup v_{7}[*] \cup v_{6}[*] \cup v_{9}[*] \cup v_{8}[*] \cup v_{4}[*] \cup v_{10}[*] \cup v_{17}[*] .
$$

Then with the sequence of pivots $v_{16}[I-b], v_{15}[I-b]$, and $v_{12}[I-b]$ we see that $P$ spreads to $v_{18}[I-b] \cup v_{14}[I-b] \cup v_{11}[I-b]$. Hence the claim follows from Example 5.9.

The cycle structure of $\Sigma$ at the vertices is the following:

$$
\begin{gathered}
v_{1}[i], v_{13}[i]: \text { cycles of length } 4|\langle-a+c+d+1\rangle| \text { and } 4|\langle 1+a\rangle|, \\
v_{2}[i], v_{4}[i]: \text { cycles of length } 4|\langle 1+a\rangle| \text { and } 3|\langle 1-c\rangle|, \\
v_{3}[i], v_{7}[i]: \text { cycles of length } 3|\langle 1-c\rangle| \text { and } 4|\langle-a+c+d+1\rangle|, \\
v_{5}[i], v_{17}[i]: \text { cycles of length } 4|\langle 1+a\rangle| \text { and } 4|\langle-b-d\rangle|, \\
v_{6}[i], v_{8}[i]: \text { cycles of length } 4|\langle-b-d\rangle| \text { and } 3|\langle 1-c\rangle|, \\
v_{9}[i], v_{10}[i]: \text { cycles of length } 4|\langle-b-d\rangle| \text { and } 4|\langle-a+c+d+1\rangle|, \\
v_{11}[i], v_{16}[i]: \text { cycles of length } 4|\langle-b-d\rangle| \text { and } 3|\langle b\rangle|, \\
v_{12}[i], v_{14}[i]: \text { cycles of length } 4|\langle-a+c+d+1\rangle| \text { and } 3|\langle b\rangle|, \\
v_{15}[i], v_{18}[i]: \text { cycles of length } 4|\langle 1+a\rangle| \text { and } 3|\langle b\rangle|,
\end{gathered}
$$


for all $i \in \mathbb{Z}_{N}$. If $b \equiv 0(\bmod N)$ and $c \equiv 1(\bmod N)$, then $\operatorname{imm}(\Sigma) \leq 5 / 18=\omega(\Sigma)$. Otherwise, using a graph isomorphism, we may assume that $b \not \equiv 0(\bmod N)$. Then $\operatorname{imm}(\Sigma) \leq(4 N+N / 2) / 18 N=1 / 4 \leq \omega(\Sigma)$.

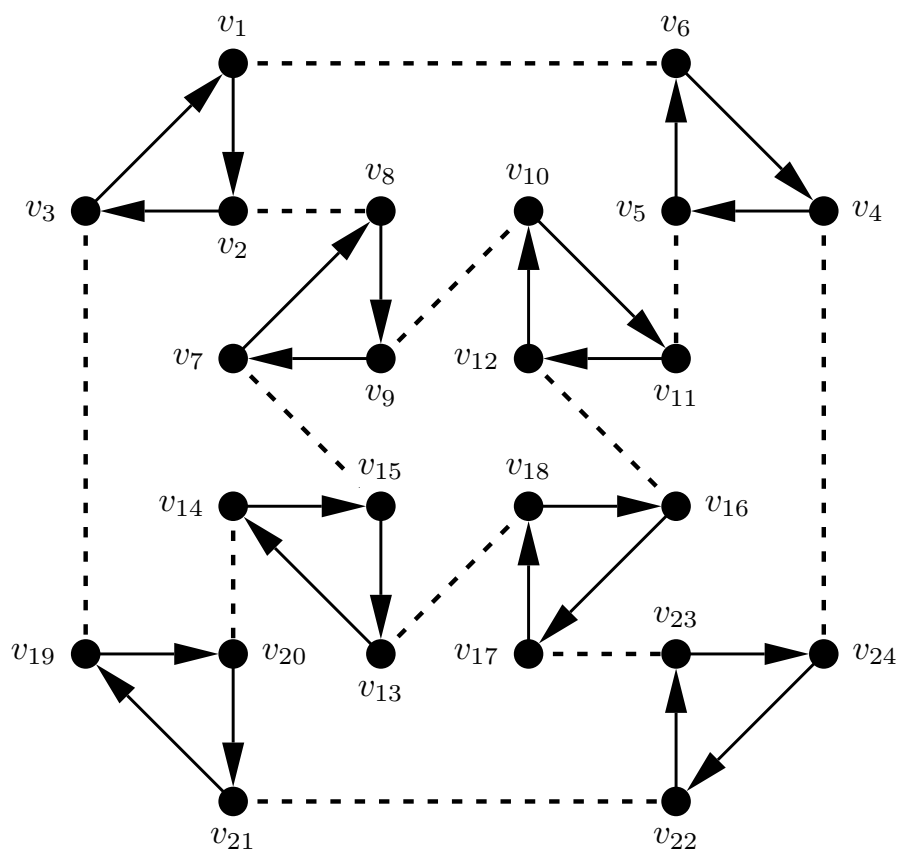

FiguRE 7.18. Schreier graph $\Sigma_{24 A}$

\subsection{The graph $\Sigma_{24 A}$.}

Lemma 7.45. Let $\Sigma$ be a covering of $\Sigma_{24 A}$ in Figure 7.18 with simply intersecting cycles. Then $\operatorname{imm}(\Sigma) \leq 1 / 4=\omega(\Sigma)$.

Proof. First we prove that $\operatorname{imm}(\Sigma) \leq 1 / 4$. A straightforward computation shows that $v_{1}[*] \cup v_{2}[*] \cup v_{3}[*] \cup v_{4}[*] \cup v_{7}[*] \cup v_{13}[*]$ is a plague. To prove that $\omega(\Sigma)=1 / 4$ observe that in every covering all cycles have length $\geq 4$.

7.19. The proof of Theorem 6.3. By Proposition 4.3, the Schreier graphs to consider are those in Figures 7.177.18. In this section we proved that only the Schreier graphs $\Sigma_{1 A}, \Sigma_{3 A}, \Sigma_{4 A}, \Sigma_{6 A}, \Sigma_{6 D}, \Sigma_{7 A}, \Sigma_{8 A}, \Sigma_{9 A}, \Sigma_{12 A}, \Sigma_{12 B}, \Sigma_{12 C}$, $\Sigma_{18 A}$, and $\Sigma_{24 A}$ have coverings with simply intersecting cycles. For each of these coverings we determined an upper bound for the immunity and then we proved that this upper bound can be bounded from above by $\omega$. The corresponding claims are Lemmas 7.2, 7.6, 7.11, 7.17, 7.26, 7.28, 7.29, 7.32, 7.33, 7.36, 7.41, 7.44, and 7.45,

\section{Nichols ALGEBRAS With MANy CUBIC RELATIONS}

We refer to 2 for an introduction to Nichols algebras and Yetter-Drinfeld modules. Some elementary facts can also be found in Section 1 . Let $\mathbb{k}$ be a field, $G$ be a group, and $V$ be an absolutely irreducible finite-dimensional Yetter-Drinfeld 
module over the group algebra $\mathbb{k} G$. Recall that $V$ decomposes as $V=\bigoplus_{x \in G} V_{x}$, where $V_{x}=\{v \in V \mid \delta(v)=x \otimes v\}$ and $\delta: V \rightarrow \mathbb{k} G \otimes V$ is the left coaction of $\mathbb{k} G$ on $V$. The support of $V$ is the set

$$
\operatorname{supp}(V)=\left\{x \in G \mid V_{x} \neq 0\right\} .
$$

It is well-known that $(V, c)$ is a braided vector space where $c \in \operatorname{Aut}(V \otimes V)$ is defined by

$$
c(u \otimes v)=g v \otimes u \quad \text { for all } u \in V_{g}, g \in \operatorname{supp} V, v \in V,
$$

and $c$ satisfies the braid relation on $V \otimes V \otimes V$.

Definition 8.1. Let $G, H$ be groups. We say that two Yetter-Drinfeld modules $V \in \mathbb{k}_{\mathbb{k} G} \mathcal{Y} \mathcal{D}, W \in{ }_{\mathbb{k} H}^{\mathbb{k} H} \mathcal{Y} \mathcal{D}$ are bg-equivalent if there exists a bijection $\varphi: \operatorname{supp} V \rightarrow$ supp $W$ and a linear isomorphism $\psi: V \rightarrow W$ such that

$$
\psi\left(V_{g}\right)=W_{\varphi(g)}, \quad \psi(g v)=\varphi(g) \psi(v)
$$

for all $g, x \in \operatorname{supp} V, v \in V$. The pair $(\psi, \varphi)$ is then called a bg-equivalence between $V$ and $W$. We also say that the Nichols algebras $\mathfrak{B}(V)$ and $\mathfrak{B}(W)$ are bg-equivalent.

The reason for $b$ and $g$ in the definition is the following.

Lemma 8.2. Let $G, H$ be groups and let $V \in \mathbb{k}_{\mathbb{k} G}^{\mathbb{k} G} \mathcal{Y} \mathcal{D}, W \in \mathbb{k}_{\mathbb{k} H}^{\mathbb{K} H} \mathcal{Y}$. If $(\psi, \varphi)$ is a bgequivalence between $V$ and $W$, then $\psi: V \rightarrow W$ is an isomorphism of braided vector spaces which maps the G-homogeneous components of $V$ to the $H$-homogeneous components of $W$.

Proof. Let $v \in V, g \in G$, and $u \in V_{g}$. Then $\psi(u) \in W_{\varphi(g)}$ and hence

$$
\begin{aligned}
(\psi \otimes \psi)(c(u \otimes v)) & =\psi(g v) \otimes \psi(u) \\
& =\varphi(g) \psi(v) \otimes \psi(u) \\
& =c(\psi(u) \otimes \psi(v)) .
\end{aligned}
$$

Thus $\psi$ is an isomorphism of braided vector spaces. The rest is clear from the assumptions on $\psi$.

In particular, Nichols algebras of bg-equivalent Yetter-Drinfeld modules are isomorphic as algebras and coalgebras. if

Recall from [6, Section 2.1] that a Nichols algebra $\mathfrak{B}(V)$ has many cubic relations

$$
\operatorname{dim} \operatorname{ker}\left(1+c_{12}+c_{12} c_{23}\right) \geq \frac{1}{3} \operatorname{dim} V\left((\operatorname{dim} V)^{2}-1\right) .
$$

Let $X=\operatorname{supp}(V), x \in X$ and $e=\operatorname{dim} V_{x}$. Assume that $X$ is an indecomposable rack of size $d>1$. Since $V=\bigoplus_{x \in X} V_{x}$, we conclude that $V^{\otimes 3}=\bigoplus_{\mathcal{O}} V_{\mathcal{O}}^{\otimes 3}$, where the direct sum is taken over all Hurwitz orbits and

$$
V_{\mathcal{O}}^{\otimes 3}=\bigoplus_{(x, y, z) \in \mathcal{O}} V_{x} \otimes V_{y} \otimes V_{z} .
$$

Each $V_{\mathcal{O}}^{\otimes 3}$ is invariant under the map $1+c_{12}+c_{12} c_{23}$.

Remark 8.3. By Theorem 6.3, the inequality $\operatorname{imm}(\mathcal{O}) \leq \omega(\mathcal{O})$ holds for $\mathcal{O} \subseteq X^{3}$ if the quotient $\overline{\mathcal{O}}$ does not have $x y$-cycles of length $\geq 5$. 
Lemma 8.4. Assume that $\mathfrak{B}(V)$ has many cubic relations. Then the following hold:

(1) $\sum_{\mathcal{O}} \operatorname{imm}(\mathcal{O}) \operatorname{dim} V_{\mathcal{O}}^{\otimes 3} \geq \frac{d e}{3}\left((d e)^{2}-1\right)$, where the sum is taken over all Hurwitz orbits of $X^{3}$.

(2) Assume that $\operatorname{imm}(\mathcal{O}) \leq \omega(\mathcal{O})$ for all $\mathcal{O} \subseteq X^{3}$. Then

$$
\sum_{\mathcal{O}} \omega(\mathcal{O}) \operatorname{dim} V_{\mathcal{O}}^{\otimes 3} \geq \frac{d e}{3}\left((d e)^{2}-1\right)
$$

Proof. (1) follows from [6, Proposition 6] and (8.1). (2) follows from (1).

Lemma 8.5. Assume that $\mathfrak{B}(V)$ has many cubic relations. Also assume that $\operatorname{imm}(\mathcal{O}) \leq \omega(\mathcal{O})$ for all $\mathcal{O} \subseteq X^{3}$. Then

$$
\sum_{p, q \geq 1} k_{p} k_{q}\left(3 \omega_{p, q}^{\prime}-1\right)+\left(\frac{1}{10}+\frac{1}{8}\right) k_{3}+\frac{1}{4} k_{4} \geq-1 .
$$

Further, if the covering $\Sigma_{6 A}^{4 ; 2,2}$ appears in $X^{3}$, then $k_{4} \geq 4$; otherwise

$$
\sum_{p, q \geq 1} k_{p} k_{q}\left(3 \omega_{p, q}^{\prime}-1\right)+\left(\frac{1}{10}+\frac{1}{8}\right) k_{3} \geq-1 .
$$

Proof. The claim on $k_{4}$ holds by Lemma 7.19)(1). We prove the first inequality of the lemma; the second is analogous. Since $\operatorname{dim} V_{\mathcal{O}}^{\otimes 3}=|\mathcal{O}| e^{3}$ and $(d e)^{2}-1 \geq(d e)^{2}-e^{2}$, we may assume that $e=1$. By Lemma 8.4(2),

$$
\sum_{v \in X^{3}} \omega(v)=\sum_{\mathcal{O}} \omega(\mathcal{O})|\mathcal{O}| \geq d\left(d^{2}-1\right) / 3
$$

By Lemmas 7.13, 7.19 and 7.42,

$$
\begin{array}{r}
\left|\left\{v \in X^{3}: v \in \Sigma_{4 A}^{5 ; 3,2}, v \in v_{1}[*]\right\}\right| \leq d k_{3}, \\
\left|\left\{v \in X^{3}: v \in \Sigma_{6 A}^{4 ; 2,2}, v \in v_{3}[*]\right\}\right| \leq d k_{4}, \\
\left|\left\{v \in X^{3}: v \in \Sigma_{12 C}^{1 ; 0,0,0}\right\}\right| \leq d k_{3} .
\end{array}
$$

By the definition of $\omega$ in (6.2), (8.2) and the last three inequalities imply that

$$
\sum_{p, q \geq 1} k_{p} k_{q} \omega_{p, q}^{\prime} d+\frac{d k_{3}}{30}+\frac{d k_{4}}{12}+\frac{d k_{3}}{24} \geq \frac{d\left(d^{2}-1\right)}{3} .
$$

Since $\sum k_{p} k_{q}=d^{2}$, the lemma follows.

Corollary 8.6. Assume that $\mathfrak{B}(V)$ has many cubic relations. Also assume that $\operatorname{imm}(\mathcal{O}) \leq \omega(\mathcal{O})$ for all $\mathcal{O} \subseteq X^{3}$. Then

$$
\left(k_{3}+k_{3}^{\prime}-5\right)^{2}+\frac{11}{5} k_{3}+k_{3}^{\prime 2} \leq 49 .
$$

Further, if the covering $\Sigma_{6 A}^{4 ; 2,2}$ appears in $X^{3}$, then $k_{4} \geq 4$; otherwise

$$
\left(k_{3}+k_{3}^{\prime}-4\right)^{2}+\frac{1}{5} k_{3}+k_{3}^{\prime 2} \leq 40 .
$$

Proof. The claim follows from Lemma 8.5 and the definition of $\omega_{p, q}^{\prime}$. Note that $k_{4} \leq k_{3}^{\prime}$. 
Remark 8.7. Recall that $k_{n} \neq 1$ for all $n \geq 3$. In [6], the case where $X$ is braided was completely classified. Thus we may assume that $X$ is not braided. Hence $k_{3}^{\prime} \geq 2$. Corollary 8.6 implies that $k_{3}^{\prime} \leq 6$. Further, if $k_{3}^{\prime}=2$, then $k_{4} \leq 2$ and $0 \leq k_{3} \leq 7$. If $k_{3}^{\prime}=3$, then $0 \leq k_{3} \leq 6$. If $k_{3}^{\prime}=4$, then $0 \leq k_{3} \leq 5$. If $k_{3}^{\prime}=5$, then $0 \leq k_{3} \leq 3$. Finally, if $k_{3}^{\prime}=6$, then $k_{3}=0$. In particular, $k_{3}+k_{3}^{\prime} \leq 9$. If the covering $\Sigma_{64}^{4 ; 2,2}$ does not appear, then we get the same solutions except $\left(k_{3}, k_{3}^{\prime}\right)=(5,4)$ and $(3,5)$.

Lemma 8.8. Assume that $\mathfrak{B}(V)$ has many cubic relations. Also assume that $\operatorname{imm}(\mathcal{O}) \leq \omega(\mathcal{O})$ for all $\mathcal{O} \subseteq X^{3}$. Then $|X| \leq 33$.

Proof. By Remark 8.7 we may assume that $\varphi_{x}$ with $x \in X$ moves at most 9 elements of $X$. There are 29 possible profiles for $\varphi_{x}$ if we ignore the number of fixed points. Using Proposition 3.2 we can exclude 11 profiles and are left with the list $1^{a} 2,1^{a} 3,1^{a} 2^{2}, 1^{a} 4,1^{a} 5,1^{a} 2^{3}, 1^{a} 24,1^{a} 3^{2}, 1^{a} 6,1^{a} 7,1^{a} 2^{4}, 1^{a} 2^{2} 4,1^{a} 26,1^{a} 4^{2}$, $1^{a} 8,1^{a} 3^{3}, 1^{a} 36,1^{a} 9$, where $a \geq 1$ is arbitrary. Theorem 2.7 says that

$$
|X| \leq\left(\sum_{j \geq 2} a_{j}\right)\left(k_{2}^{\prime}-2\right)+k_{2}^{\prime}+1 .
$$

Since $k_{2}^{\prime} \leq 9$ is the number of points moved by $\varphi_{x}, x \in X$, we conclude that $|X| \leq 31$ if $\sum_{j \geq 2} a_{j} \leq 3$. The only profile not satisfying the latter inequality is $1^{a} 2^{4}$. For this we get $|X| \leq 33$. This yields the claim.

Lemma 8.9. Assume that $\mathfrak{B}(V)$ has many cubic relations and the rack $X$ satisfies $k_{3}^{\prime}(X) \neq 0$. Also assume that $\operatorname{imm}(\mathcal{O}) \leq \omega(\mathcal{O})$ for all $\mathcal{O} \subseteq X^{3}$. Then $X$ is isomorphic to $\mathbb{D}_{5}, \mathbb{D}_{7}, \operatorname{Aff}(5,2)$, $\operatorname{Aff}(5,3)$, $\operatorname{Aff}(7,2)$ or $\operatorname{Aff}(7,4)$.

Remark 8.10. In fact, in Lemma 8.9 the rack $X$ has to be isomorphic to $\operatorname{Aff}(5,2)$ or $\operatorname{Aff}(5,3)$. The proof of this fact is more complicated and it is not needed here.

Proof. By Lemma 8.8, $|X| \leq 33$. Since indecomposable quandles of size $\leq 35$ were classified in [10], a straightforward computer calculation gives a complete list of indecomposable non-braided injective quandles satisfying (8.3). We obtain the list given in the lemma. The following table contains some properties of these racks:

\begin{tabular}{c|c|c} 
Quandle & Sizes of orbits & Remark \\
\hline $\mathbb{D}_{5}$ & 1,24 & \\
$\operatorname{Aff}(5,2)$ and $\operatorname{Aff}(5,3)$ & 1,24 & \\
$\mathbb{D}_{7}$ & 1,48 & $k_{7}=6$ \\
$\operatorname{Aff}(7,2)$ and $\operatorname{Aff}(7,4)$ & $1,42,49$ &
\end{tabular}

This completes the proof.

\section{Computation of the 2-COCyCles And the Main theORem}

Let $X$ be one of the racks $\operatorname{Aff}(5,2)$ or $\operatorname{Aff}(5,3)$. Recall that $G_{X}$ is the enveloping group of $X$. For all $i \in X$ let $x_{i}$ be the image of $i$ under the canonical map $X \rightarrow G_{X}$.

Lemma 9.1 ([5, Lemma 5.4]). The centralizer of $x_{1}$ in $G_{X}$ is the cyclic group generated by $x_{1}$. 
TABLE 9.1. Finite-dimensional elementary Nichols algebras

\begin{tabular}{|l|l|l|l|l|}
\hline Rack & Rank & Dimension & Hilbert series & Remark \\
\hline$\{1\}$ & 1 & $n \geq 2$ & $(n)_{t}$ & \\
\hline$(12)^{\mathbb{S}_{3}}$ & 3 & 12 & $(2)_{t}^{2}(3)_{t}$ & \\
\hline$(12)^{\mathbb{S}_{3}}$ & 3 & 432 & $(3)_{t}(4)_{t}(6)_{t}(6)_{t^{2}}$ & char $\mathbb{k}=2$ \\
\hline$(123)^{\mathbb{A}_{3}}$ & 4 & 36 & $(2)_{t}^{2}(3)_{t}^{2}$ & char $\mathbb{k}=2$ \\
\hline$(123)^{\mathbb{A}_{4}}$ & 4 & 72 & $(2)_{t}^{2}(3)_{t}(6)_{t}$ & char $\mathbb{k} \neq 2$ \\
\hline$(123)^{\mathbb{A}_{4}}$ & 4 & 5184 & $(6)_{t}^{4}(2)_{t^{2}}^{2}$ & \\
\hline$(12)^{\mathbb{S}_{4}}$ & 6 & 576 & $(2)_{t}^{2}(3)_{t}^{2}(4)_{t}^{2}$ & \\
\hline$(12)^{\mathbb{S}_{4}}$ & 6 & 576 & $(2)_{t}^{2}(3)_{t}^{2}(4)_{t}^{2}$ & \\
\hline$(1234)^{\mathbb{S}_{4}}$ & 6 & 576 & $(2)_{t}^{2}(3)_{t}^{2}(4)_{t}^{2}$ & \\
\hline $\operatorname{Aff}(5,2)$ & 5 & 1280 & $(4)_{t}^{4}(5)_{t}$ & \\
\hline $\operatorname{Aff}(5,3)$ & 5 & 1280 & $(4)_{t}^{4}(5)_{t}$ & \\
\hline $\operatorname{Aff}(7,3)$ & 7 & 326592 & $(6)_{t}^{6}(7)_{t}$ & \\
\hline $\operatorname{Aff}(7,5)$ & 7 & 326592 & $(6)_{t}^{6}(7)_{t}$ & \\
\hline$(12)^{\mathbb{S}_{5}}$ & 10 & 8294400 & $(4)_{t}^{4}(5)_{t}^{2}(6)_{t}^{4}$ & \\
\hline$(12)^{\mathbb{S}_{5}}$ & 10 & 8294400 & $(4)_{t}^{4}(5)_{t}^{2}(6)_{t}^{4}$ & \\
\hline
\end{tabular}

Let $\rho$ be an absolutely irreducible representation of $C_{G_{X}}\left(x_{1}\right)$. By straightforward but lengthy calculations we obtain the following.

Lemma 9.2. Let $\mathcal{O} \subseteq X^{3}$ be a Hurwitz orbit of size 24. Then

$$
\left.\operatorname{dim} \operatorname{ker}\left(1+c_{12}+c_{12} c_{23}\right)\right|_{V_{\mathcal{O}}^{\otimes 3}} \leq \begin{cases}8 & \text { if } \rho\left(x_{1}\right)=-1 \\ 5 & \text { otherwise. }\end{cases}
$$

Theorem 9.3. Let $G$ be a group, $V$ be a finite-dimensional absolutely irreducible Yetter-Drinfeld module over $G$, and $X=\operatorname{supp} V$. Assume that $X$ is an indecomposable rack such that no quotient of a Hurwitz orbit in $X^{3}$ contains an xy-cycle of length $\geq 5$. Also assume that $\mathfrak{B}(V)$ has many cubic relations.

(1) The rack $X$ is isomorphic to one of the following:

$$
\begin{gathered}
(12)^{\mathbb{S}_{n}} \text { for } n \in\{3,4,5\}, \\
(1234)^{\mathbb{S}_{4}},(123)^{\mathbb{A}_{4}}, \\
\operatorname{Aff}(p, \alpha) \text { for }(p, \alpha) \in\{(5,2),(5,3),(7,3),(7,5)\} .
\end{gathered}
$$

(2) The Nichols algebra $\mathfrak{B}(V)$ is bg-equivalent to one of the Nichols algebras listed in Table 9.1 .

(3) The Hilbert series of $\mathfrak{B}(V)$ is t-integral of depth two.

Remark 9.4. Table 9.1 does not contain the information on the 2-cocycle of $X$ corresponding to $V$. For the racks $\operatorname{Aff}(5,2)$ and $\operatorname{Aff}(5,3)$ we use the constant 2cocycle -1 ; see the proof of Theorem 9.3 . For the others, see [6, Appendix A].

Proof. In [6, Theorem 11] the theorem was proved under the additional assumption that $X$ is braided. One obtains the same racks except $\operatorname{Aff}(5,2)$ and $\operatorname{Aff}(5,3)$. Assume now that $X$ is not braided, i.e. $k_{3}^{\prime}(X) \neq 0$. By Lemma 8.9, $X$ is one of the racks $\operatorname{Aff}(5,2), \operatorname{Aff}(5,3), \mathbb{D}_{5}, \mathbb{D}_{7}, \operatorname{Aff}(7,2)$ and $\operatorname{Aff}(7,4)$. The racks $\mathbb{D}_{5}, \mathbb{D}_{7}$, $\operatorname{Aff}(7,2)$ and $\operatorname{Aff}(7,4)$ can be excluded since they have $x y$-cycles of length $\geq 5$ in some Hurwitz orbit quotients. 
TABLE 9.2. Some small racks

\begin{tabular}{|c|c|c|c|}
\hline Rack & Size of orbits & Number & Bound for immunity \\
\hline $\mathbb{D}_{5}$ & 1 & 5 & 1 \\
& 24 & 5 & $7 / 24$ \\
\hline $\mathbb{D}_{7}$ & 1 & 7 & 1 \\
& 48 & 7 & $14 / 48$ \\
\hline Aff $(7, \alpha)$ & 1 & 1 & 1 \\
$\alpha=2,4$ & 42 & 1 & $9 / 42$ \\
& 49 & 6 & $15 / 49$ \\
\hline
\end{tabular}

Now let $X=\operatorname{Aff}(5,2)$ or $X=\operatorname{Aff}(5,3)$. Then $X^{3}$ decomposes into five Hurwitz orbits of size 1 and five of size 24. Since $\mathfrak{B}(V)$ has many cubic relations, (8.1) and Lemma 9.2 imply that $\rho\left(x_{1}\right)=-1$. Moreover, in this case (8.1) holds and the Nichols algebras of these $V$ are known to be finite-dimensional; see e.g. 5 , Proposition 5.15]. The claim on the Hilbert series is known for all Nichols algebras in Table 9.1 .

Remark 9.5. In Theorem 9.3 the assumption about the length of an $x y$-cycle in the quotient of a Hurwitz orbit in $X^{3}$ can be replaced by the following weaker assumption (see Theorem 6.3):

$$
\operatorname{imm}(\mathcal{O}) \leq \omega(\mathcal{O})
$$

for all Hurwitz orbits $\mathcal{O}$ of $X^{3}$. To prove this, we exclude the racks $\mathbb{D}_{5}, \mathbb{D}_{7}, \operatorname{Aff}(7,2)$ and Aff $(7,4)$. Straightforward calculations yield the information listed in Table 9.2 . Hence the inequality of Lemma 8.4(1) cannot be satisfied for the racks $\mathbb{D}_{7}, \operatorname{Aff}(7,2)$ and $\operatorname{Aff}(7,4)$. The rack $\mathbb{D}_{5}$ cannot be excluded with this argument. However, it can be excluded by computing

$$
\left.\operatorname{dim} \operatorname{ker}\left(1+c_{12}+c_{12} c_{23}\right)\right|_{V_{\mathcal{O}}^{\otimes 3}},
$$

where $\mathcal{O}$ is an orbit of size 24 . This is essentially what we did for the racks $\operatorname{Aff}(5,2)$ and $\operatorname{Aff}(5,3)$.

We conclude the paper with the following conjecture.

Conjecture 9.6. The inequality $\operatorname{imm}(\Sigma) \leq \omega(\Sigma)$ holds for all $\mathbb{B}_{3}$-spaces $\Sigma$ with simply intersecting cycles.

According to Remark 9.5. Conjecture 9.6 implies Conjecture 1.1 formulated in the introduction.

\section{ACKNOWLEDGEMENTS}

The authors are grateful to the referee for pointing out some typos and for asking for clarification of some proofs. GAP (http://www.gap-system.org/) and Rig (http://code.google.com/p/rig/) were used for some computations.

\section{REFERENCES}

[1] Nicolás Andruskiewitsch and Matías Graña, From racks to pointed Hopf algebras, Adv. Math. 178 (2003), no. 2, 177-243. MR1994219(2004i:16046) 
[2] Nicolás Andruskiewitsch and Hans-Jürgen Schneider, Pointed Hopf algebras, New directions in Hopf algebras, Math. Sci. Res. Inst. Publ., vol. 43, Cambridge Univ. Press, Cambridge, 2002, pp. 1-68. MR.1913436 (2003e:16043)

[3] E. Brieskorn, Automorphic sets and braids and singularities, Braids (Santa Cruz, CA, 1986), Contemp. Math., vol. 78, Amer. Math. Soc., Providence, RI, 1988, pp. 45-115. MR975077 (90a:32024)

[4] Tullio Ceccherini-Silberstein and Michel Coornaert, Cellular automata and groups, Springer Monographs in Mathematics, Springer-Verlag, Berlin, 2010. MR2683112 (2011j:37002)

[5] M. Graña, I. Heckenberger, and L. Vendramin, Nichols algebras of group type with many quadratic relations, Adv. Math. 227 (2011), no. 5, 1956-1989. MR2803792 (2012f:16077)

[6] I. Heckenberger, A. Lochmann, and L. Vendramin, Braided racks, Hurwitz actions and Nichols algebras with many cubic relations, Transform. Groups 17 (2012), no. 1, 157-194. MR2891215

[7] A. Hurwitz, Ueber Riemann'sche Flächen mit gegebenen Verzweigungspunkten, Math. Ann. 39 (1891), no. 1, 1-60. MR1510692

[8] Christian Kassel and Vladimir Turaev, Braid groups, with the graphical assistance of Olivier Dodane. Graduate Texts in Mathematics, vol. 247, Springer, New York, 2008. MR2435235 (2009e:20082)

[9] Robert A. Rankin, Modular forms and functions, Cambridge University Press, Cambridge, 1977. MR0498390 (58:16518)

[10] L. Vendramin, On the classification of quandles of low order, J. Knot Theory Ramifications 21 (2012), no. 9, 1250088, 10. MR2926571

FB Mathematik und Informatik, Philipps-Universität Marburg, Hans-MeerweinStrasse, 35032 Marburg, Germany

E-mail address: heckenberger@mathematik.uni-marburg.de

FB Mathematik und Informatik, Philipps-Universität Marburg, Hans-MeerweinStrasse, 35032 Marburg, Germany

E-mail address: lochmann@mathematik.uni-marburg.de

FB Mathematik und Informatik, Philipps-Universität Marburg, Hans-MeerweinStrasse, 35032 Marburg, Germany

E-mail address: lvendramin@dm.uba.ar 